\title{
ALGEBRAICALLY RIGID SIMPLICIAL COMPLEXES AND GRAPHS
}

\author{
KLAUS ALTMANN, MINA BIGDELI, JÜRGEN HERZOG AND DANCHENG LU
}

\begin{abstract}
We call a simplicial complex algebraically rigid if its Stanley-Reisner ring admits no nontrivial infinitesimal deformations, and call it inseparable if it does not allow any deformation to other simplicial complexes. Algebraically rigid simplicial complexes are inseparable. In this paper we study inseparability and rigidity of Stanley-Reisner rings, and apply the general theory to letterplace ideals as well as to edge ideals of graphs. Classes of algebraically rigid simplicial complexes and graphs are identified.
\end{abstract}

\section{INTRODUCTION}

In the study of monomial ideals it is a popular technique to polarize in order to obtain squarefree monomial ideals. Let $S=K\left[x_{1}, \ldots, x_{n}\right]$ be the polynomial ring in $n$ indeterminates over the field $K$. Given a monomial ideal $I \subseteq S$, the polarized ideal $I^{\wp}$ of $I$ is a squarefree monomial ideal defined in a larger polynomial ring $S^{\wp}$ and $S / I$ is obtained from $S^{\wp} / I^{\wp}$ by reduction modulo a regular sequence of linear forms consisting of differences of variables, see [7, page 19] for details. In other words, $S^{\wp} / I^{\wp}$ may be viewed an unobstructed deformation of $S / I$ over a suitable affine space.

The natural question arises whether $S^{\wp} / I^{\wp}$ or any other $K$-algebra defined by a squarefree monomial ideal admits further unobstructed deformations, or at least non-trivial infinitesimal deformations. This may be indeed the case as the third author learned from Fløystad.

Separation. Let $I \subseteq S$ be a squarefree monomial ideal, and let $y$ be an indeterminate over $S$. Fløystad (see [4]) calls a monomial ideal $J \subseteq S[y]$ a separation of $I$ for the variable $x_{i}$ if the following conditions hold:

(i) the ideal $I$ is the image of $J$ under the $K$-algebra homomorphism $S[y] \rightarrow S$ with $y \mapsto x_{i}$ and $x_{j} \mapsto x_{j}$ for all $1 \leq j \leq n$;

(ii) $x_{i}$ and $y$ divide some minimal generators of $J$;

(iii) $y-x_{i}$ is a non-zero divisor of $S[y] / J$.

The ideal $I$ is called separable if it admits a separation, otherwise it is called inseparable.

2010 Mathematics Subject Classification. Primary 13D10, 13C13; Secondary 05E40.

Key words and phrases. infinitesimal deformations, rigidity, Stanley-Reisner ideals, edge ideals.

The paper was written while the second and the fourth authors were visiting the Department of Mathematics of University Duisburg-Essen. They want to express their thanks for the hospitality.

Corresponding author: Dancheng Lu. 
If $J \subseteq S[y]$ is a separation of $I$ for the variable $x_{i}$, then $K[t] \rightarrow S[y] / J, t \mapsto y-x_{i}$, is a non-trivial unobstructed deformation of $S / I$ over $\mathbb{A}^{1}=\operatorname{Spec} K[t]$. The simplest example of a squarefree monomial ideal which admits a separation is the ideal $I=$ $\left(x_{1} x_{2}, x_{1} x_{3}, x_{2} x_{3}\right)$. One possible separation of $I$ is the ideal $J=\left(x_{1} y, x_{1} x_{3}, x_{2} x_{3}\right)$.

Infinitesimal deformations. But even if $I \subseteq S$ is inseparable, it still may admit infinitesimal deformations. We denote by $\varepsilon$ a non-zero term with $\varepsilon^{2}=0$. Let $J \subseteq S[\varepsilon]$ be an ideal. Then $S[\varepsilon] / J$ is called an infinitisimal deformation of $S / I$ if the canonical $K$-algebra homomorphism $K[\varepsilon] \rightarrow S[\varepsilon] / J$ is flat and if $S / I$ is obtained from $S[\varepsilon] / J$ by reduction modulo $\varepsilon$. Thus if $I=\left(f_{1}, \ldots, f_{m}\right)$, then $J=\left(f_{1}+\right.$ $\left.g_{1} \varepsilon, \ldots, f_{m}+g_{m} \varepsilon\right)$ and $K[\varepsilon] \rightarrow S[\varepsilon] / J$ is flat if and only if $\varphi: I \rightarrow S / I$ with $f_{i} \mapsto$ $g_{i}+I$ is a well-defined $S$-module homomorphism. In other words, the infinitesimal deformations of $S / I$ are in bijection to the elements of $I^{*}=\operatorname{Hom}_{S}(I, S / I)$.

Cotangent functors. Recall that a $K$-linear map $\partial: S \rightarrow S$ is called a $K$-derivation if $\partial f g=f \partial g+g \partial f$. The set of $K$-derivations has a natural structure as an $S$ module, and is denoted $\operatorname{Der}_{K}(S)$. In fact, $\operatorname{Der}_{K}(S)$ is a free $S$-module whose basis consists of the partial derivatives $\partial / \partial x_{i}$. The infinitesimal deformation $S[\varepsilon] / J$ of $S / I$ is called trivial if there exists a $K$-algebra automorphism $S[\varepsilon] \rightarrow S[\varepsilon]$ which maps $J$ to $I S[\varepsilon]$. This is the case, if and only if there exists $\partial \in \operatorname{Der}_{K}(S)$ such that $J=\left(f_{1}+\partial f_{1} \varepsilon, \ldots, f_{m}+\partial f_{m} \varepsilon\right)$. Thus if we consider the natural map $\delta^{*}: \operatorname{Der}_{K}(S) \rightarrow$ $I^{*}$ which assigns to $\partial \in \operatorname{Der}_{K}(S)$ the element $\delta^{*}(\partial)$ with $\delta^{*}(\partial)\left(f_{i}\right)=\partial f_{i}+I$, then the non-zero elements of Coker $\delta^{*}$ are in bijection to the isomorphism classes of non-trivial infinitesimal deformations of $S / I$, see for example [6, Lemma 2.4]. This cokernel is denoted by $T^{1}(S / I)$ and is called the first cotangent functor of $S / I$. The cotangent functors have been first introduced by Lichtenbaum and Schlessinger [9]. The $K$-algebra $S / I$ is called rigid if it does not admit any non-trivial infinitesimal deformations. Hence $S / I$ is rigid if and only if $T^{1}(S / I)=0$. For simplicity we call $I$ rigid if $S / I$ is rigid. The simplest example of a squarefree monomial ideal which is inseparable but not rigid, is the ideal $I=\left(x_{1} x_{2}, x_{2} x_{3}, x_{3} x_{4}\right)$, see Proposition 3.7(a) and Theorem 3.10. We recommend the reader to consult [10, Section 3] for general basic facts about deformation theory.

Rigidity of simplicial complexes. In the case that $I \subseteq S$ is a monomial ideal, $T^{1}(S / I)$ is a $\mathbb{Z}^{n}$-graded $S$-module. If moreover $I$ is a squarefree monomial ideal, then the $\mathbb{Z}^{n}$-graded components of $T^{1}(S / I)$ have a combinatorial interpretation as was shown by the first author and Christophersen in [2] and [1]. We recall some of these results in Section 1 of this paper because they are crucial for later applications.

Recall that a simplicial complex $\Delta$ on the vertex set $V$ is a collection of subset of $V$ such that whenever $F \in \Delta$ and $G \subset F$, then $G \in \Delta$. Frequently we denote the vertex set of $\Delta$ by $V(\Delta)$.

Since $I$ is a squarefree monomial ideal, there exists a unique simplicial complex $\Delta$ such that $I=I_{\Delta}$, where $I_{\Delta}$ is the Stanley-Reisner ideal of $\Delta$. As usual, $S / I_{\Delta}$ is denoted by $K[\Delta]$ and is called the Stanley-Reisner ring of $\Delta$. For simplicity we will write $T^{1}(\Delta)$ for $T^{1}(K[\Delta])$. We say that $\Delta$ is algebraically rigid (with respect to $K$ ) if $K[\Delta]$ is rigid. Actually it is shown in Section 1 that algebraic rigidity of simplicial 
complexes does not depend on $K$. There is the concept of rigid simplicial complexes, meaning that the simplicial complex does not admit any non-trivial automorphism. In this paper rigidity means algebraic rigidity, and will simply say that $\Delta$ is rigid if it is algebraically rigid.

Description of contents. The aim of this paper is to characterize rigid simplicial complexes in combinatorial terms and exhibit classes of them. By what we said before, it follows that $\Delta$ is rigid if and only if $T^{1}(\Delta)_{\mathbf{c}}=0$ for all $\mathbf{c} \in \mathbb{Z}^{n}$. The important facts, shown in [2], regarding the graded components of $T^{1}(\Delta)$ that will be used throughout the paper, are the following: write $\mathbf{c}=\mathbf{a}-\mathbf{b}$ with $\mathbf{a}, \mathbf{b} \in \mathbb{N}^{n}$ and $\operatorname{supp} \mathbf{a} \cap \operatorname{supp} \mathbf{b}=\emptyset$. Here for a vector $\mathbf{a}, \operatorname{supp} \mathbf{a}$ is defined to be the set $\left\{i \in[n]: a_{i} \neq 0\right\}$ where the $a_{i}$ are the components of $\mathbf{a}$. Then

(i) $T^{1}(\Delta)_{\mathbf{a}-\mathbf{b}}=0$ if $\mathbf{b} \notin\{0,1\}^{n}$, and if $\mathbf{b} \in\{0,1\}^{n}$, then $T^{1}(\Delta)_{\mathbf{a}-\mathbf{b}}$ depends only on supp a and supp b;

(ii) $T^{1}(\Delta)_{\mathbf{a}-\mathbf{b}}=T^{1}\left(\operatorname{link}_{\Delta} \operatorname{supp} \mathbf{a}\right)_{-\mathbf{b}}$.

We say that $\Delta$ is $\emptyset$-rigid if $T^{1}(\Delta)_{-\mathbf{b}}=0$ for all $\mathbf{b} \in\{0,1\}^{n}$. Thus, by (ii), $\Delta$ is rigid, if and only if all its links are $\emptyset$-rigid. These and other facts are recalled in Section 1. We close the section by applying the general theory to characterize inseparable simplicial complexes. Say, $\Delta$ is a simplicial complex on the vertex set $[n]$. To each vertex $i$ of $\Delta$ one attaches a graph $G_{\{i\}}(\Delta)$ whose vertices are those faces $F \in \Delta$ for which $F \cup\{i\} \notin \Delta$. The edges of $G_{\{i\}}(\Delta)$ are those $\{F, G\}$ for which $F \subsetneq G$ or $G \subsetneq F$. In Theorem 1.8 we show that $\Delta$ is inseparable if and only if $G_{\{i\}}(\Delta)$ is connected for $i=1, \ldots, n$, and that this is equivalent to the condition that $T^{1}(\Delta)_{-\mathbf{e}_{i}}=0$ for $i=1, \ldots, n$. Here $\mathbf{e}_{i}$ denotes the $i$ th canonical basis vector of $\mathbb{Z}^{n}$.

Let $\operatorname{dim}_{K} T^{1}(\Delta)_{-\mathbf{e}_{i}}=k$. Iterating simple separation steps one can construct a simplicial complex $\widetilde{\Delta}$ on the vertex set $([n] \backslash\{i\}) \cup\left\{v_{0}, v_{1}, \ldots, v_{k}\right\}$ defined as $k$ separation of $\Delta$ for the vertex $i$ having the property that $T^{1}(\widetilde{\Delta})_{-\mathbf{b}}=0$ for all $\mathbf{b}$ with supp $\mathbf{b} \subseteq\left\{v_{0}, \ldots, v_{k}\right\}$, and such that $K[\Delta]$ is obtained from $K[\widetilde{\Delta}]$ by cutting down by a regular sequence consisting of differences of variables.

In Section 2 we consider various operations on simplicial complexes and study their behaviour with respect to rigidity. In Proposition 2.3 it is shown that the join $\Delta_{1} * \Delta_{2}$ of the simplicial complexes $\Delta_{1}$ and $\Delta_{2}$ is rigid if and only if this is the case for $\Delta_{1}$ and $\Delta_{2}$.

More complicated is the situation for the disjoint union of two simplicial complexes $\Delta_{1}$ and $\Delta_{2}$. Here we assume that none of the two simplicial complexes is the empty set and that their 0-dimensional faces correspond to their vertex set, a condition that we do not require in general. Under these (very weak) assumptions it is shown in Theorem 2.5 that $\Delta_{1} \cup \Delta_{2}$ is inseparable if and only if $\Delta_{1}$ and $\Delta_{2}$ are simplices, and that $\Delta_{1} \cup \Delta_{2}$ is rigid if and only in addition one of the simplices has positive dimension. As a consequence we see that a disconnected simplicial complex of positive dimension is never rigid, unless all its components are simplices.

Finally in Theorem 2.10 we consider what we call the circ of two simplicial complexes, denoted by $\Delta_{1} \circ \Delta_{2}$. Suppose that $V_{i}$ is the vertex set of $\Delta_{i}$ and 
that $V_{1} \cap V_{2}=\emptyset$. Then, by definition, $F \subseteq V_{1} \cup V_{2}$ is a face of $\Delta_{1} \circ \Delta_{2}$ if and only if either $F \cap V_{1}$ is a face of $\Delta_{1}$ or $F \cap V_{2}$ is a face of $\Delta_{2}$. Note that $\Delta_{1} \circ \Delta_{2}=\left(\Delta_{1} *\left\langle V_{2}\right\rangle\right) \cup\left(\left\langle V_{1}\right\rangle * \Delta_{2}\right)$ and that $I_{\Delta_{1} \circ \Delta_{2}}=\left(I_{\Delta_{1}} I_{\Delta_{2}}\right)$. It turns out that $\Delta_{1}$ and $\Delta_{2}$ are rigid if $\Delta_{1} \circ \Delta_{2}$ is rigid and $I_{\Delta_{1}}, I_{\Delta_{2}} \neq 0$. The converse is true only under some additional assumptions.

A motivation for us to study the circ-operation resulted from the desire to classify the rigid letterplace ideals, see [4]. Given two finite posets $\mathcal{P}$ and $\mathcal{Q}$, one assigns a monomial ideal $L(\mathcal{P}, \mathcal{Q})$, which in the case $\mathcal{P}=[n]$ or $\mathcal{Q}=[n]$ is called a letterplace ideal or a co-letterplace ideal, respectively. Letterplace and co-letterplace ideals have been considered before in [3]. In the paper [4] it is shown that all letterplace ideals are inseparable. Here we show that $L(\mathcal{P}, \mathcal{Q})$ is rigid if and only if no two elements of $\mathcal{P}$ are comparable, see Theorem 2.12. In the proof of one direction of this theorem we need the circ-construction.

The last section of this paper is concerned with the rigidity of edge ideals. Given a finite simple graph on the vertex set $[n]$ one assigns to it the so-called edge ideal $I(G)$ generated by the monomials $x_{i} x_{j}$ with $\{i, j\}$ an edge of $G$. Obviously $I(G)=I_{\Delta(G)}$ for some simplicial complex $\Delta(G)$. This simplicial complex is called the independence complex of $G$. Indeed, its faces are the independent sets of $G$, that is, the subsets of $[n]$ which do not contain any edge of $G$. We say that $G$ is rigid if $\Delta(G)$ is rigid. Again there exist already various concepts of rigid graphs which should not be confused with the definition of rigidity used in this paper. Similarly, we say that $G$ is inseparable if $I(G)$ is inseparable. The ultimate goal would be to classify all rigid and inseparable graphs. It is not clear whether a nice description of these classes of graphs is possible. However with some additional assumptions on the graphs, inseparable or rigid graphs can be characterized combinatorially. Recall that a vertex $i$ of $G$ is called a free vertex if it belongs to only one edge, and an edge is called a leaf if it has a free vertex. Finally an edge $e$ of $G$ is called a branch, if there exists a leaf $e^{\prime}$ with $e^{\prime} \neq e$ such that $e \cap e^{\prime} \neq \emptyset$. Our main result on rigidity of graphs is formulated in Theorem [3.10: Let $G$ be a graph on the vertex set $[n]$ such that $G$ does not contain any induced cycle of length 4,5 or 6 . Then $G$ is rigid if and only if each edge of $G$ is a branch and each vertex of a 3-cycle of $G$ belongs to a leaf. Theorem 3.10 has several consequences. In Corollary 3.11 it is shown that a chordal graph $G$ is rigid if and only if each edge of $G$ is a branch and each vertex of a 3-cycle of $G$ belongs to a leaf. Another consequence is the fact that a graph with the property that all cycles have length $\geq 7$ is rigid if and only if each of its edges is a branch, see Corollary 3.12. This result implies in particular that a forest consisting only of branches is rigid. Finally we notice in Corollary 3.13 that a cycle is rigid if and only if it is a 4- or 6-cycle.

\section{The COtAngent FUnCtor $T^{1}$ And Rigid AND InSEPARABLE STANLEY-REISNER RINGS}

The cotangent functor $T^{1}$. Let $\Delta$ be a simplicial complex on the vertex set $V(\Delta)=$ $[n]$ where $[n]=\{1, \ldots, n\}$. We denote by $[\Delta]$ the set of elements $i \in[n]$ with $\{i\} \in \Delta$. Let $F_{1}, \ldots, F_{m} \subseteq[n]$. We denote by $\left\langle F_{1}, \ldots, F_{m}\right\rangle$ the smallest simplicial 
complex $\Delta$ with $F_{i} \in \Delta$ for $i=1, \ldots, m$. The elements of $\Delta$ are called faces. A facet of $\Delta$ is a face of $\Delta$ which is maximal with respect to inclusion. The set of facets of $\Delta$ will be denoted by $\mathcal{F}(\Delta)$.

We fix a field $K$. The ideal $I_{\Delta}$ denotes the Stanley-Reisner ideal in $S=K\left[x_{1}, \ldots, x_{n}\right]$, that is, the ideal generated by the monomial $x_{N}$ with $N \subseteq[n]$ a non-face of $\Delta$. Here $x_{N}=\prod_{i \in N} x_{i}$. The $K$-algebra $K[\Delta]=S / I_{\Delta}$ is called the Stanley-Reisner ring of $\Delta$.

The cotangent cohomology modules $T^{i}(K[\Delta])$ which we denote by $T^{i}(\Delta)$ are $\mathbb{Z}^{n_{-}}$ graded. We quote several facts about the $\mathbb{Z}^{n}$-graded components of $T^{i}(\Delta)$ which were shown in [2].

We write $\mathbf{c} \in \mathbb{Z}^{n}$ as $\mathbf{a}-\mathbf{b}$ with $\mathbf{a}, \mathbf{b} \in \mathbb{N}^{n}$ and $\operatorname{supp} \mathbf{a} \cap \operatorname{supp} \mathbf{b}=\emptyset$, and set $A=\operatorname{supp} \mathbf{a}$ and $B=\operatorname{supp} \mathbf{b}$. Here $\mathbb{N}$ denotes the set of non-negative integers, and as in the introduction the support of a vector $\mathbf{a} \in \mathbb{N}^{n}$ is defined to be the set $\operatorname{supp} \mathbf{a}=\left\{i \in[n]: a_{i} \neq 0\right\}$.

Theorem 1.1 ([2], Theorem 9). (a) $T^{i}(\Delta)_{\mathbf{a}-\mathbf{b}}=0$ if $\mathbf{b} \notin\{0,1\}^{n}$.

(b) Assuming $\mathbf{b} \in\{0,1\}^{n}, T^{i}(\Delta)_{\mathbf{a}-\mathbf{b}}$ depends only on $A$ and $B$.

Recall that for a subset $A$ of $[n]$, the link of $A$ is defined to be

$$
\operatorname{link}_{\Delta} A=\{F \in \Delta: F \cap A=\emptyset, F \cup A \in \Delta\}
$$

with vertex set $V\left(\operatorname{link}_{\Delta} A\right)=[n] \backslash A$.

We will also need the following result:

Proposition 1.2 ([2], Proposition 11). (a) $T^{i}(\Delta)_{\mathbf{a}-\mathbf{b}}=0$, unless

$$
A \in \Delta \text { and } \emptyset \neq B \subseteq\left[\operatorname{link}_{\Delta} A\right] \text {. }
$$

(b) $T^{i}(\Delta)_{\mathbf{a}-\mathbf{b}}=T^{i}\left(\operatorname{link}_{\Delta} A\right)_{-\mathbf{b}}$.

In the present paper, we are only interested in $T^{1}$. Because of Proposition 1.2(b) it is important to know how to compute $T^{1}(\Delta)_{-\mathbf{b}}$ for $B \subseteq[\Delta]$. For this purpose we introduce some notation.

Let $\mathcal{Y}$ be a collection of subsets of $[n]$. We set $\mathcal{K}^{0}(\mathcal{Y})=\{\lambda: \mathcal{Y} \rightarrow K\}$ and

$$
\mathcal{K}^{1}(\mathcal{Y})=\left\{\lambda:\left\{\left(Y_{0}, Y_{1}\right) \in \mathcal{Y}^{2}: Y_{0} \cup Y_{1} \in \mathcal{Y}\right\} \rightarrow K\right\}
$$

and define the $K$-linear map $d: \mathcal{K}^{0}(\mathcal{Y}) \rightarrow \mathcal{K}^{1}(\mathcal{Y})$ by $(d \lambda)\left(Y_{0}, Y_{1}\right)=\lambda\left(Y_{1}\right)-\lambda\left(Y_{0}\right)$.

Next given $B \subseteq[n]$ and $\Delta$, we define

$$
\begin{aligned}
& N_{B}(\Delta)=\{F \in \Delta: F \cap B=\emptyset, F \cup B \notin \Delta\}, \\
& \widetilde{N}_{B}(\Delta)=\left\{F \in N_{B}(\Delta): \text { there exists } B^{\prime} \subsetneq B \text { with } F \cup B^{\prime} \notin \Delta\right\} .
\end{aligned}
$$

With the notation introduced one has

Proposition 1.3 ([2], Corollary 6). (a) Suppose $|B| \geq 2$. Then

$$
T^{1}(\Delta)_{-\mathbf{b}}=\operatorname{Ker}\left(\mathcal{K}^{0}\left(N_{B}(\Delta)\right) \stackrel{(d, r)}{\longrightarrow} \mathcal{K}^{1}\left(N_{B}(\Delta)\right) \oplus \mathcal{K}^{0}\left(\tilde{N}_{B}(\Delta)\right)\right),
$$

where $d: \mathcal{K}^{0}\left(N_{B}(\Delta)\right) \rightarrow \mathcal{K}^{1}\left(N_{B}(\Delta)\right)$ is the map as defined above and $r: \mathcal{K}^{0}\left(N_{B}(\Delta)\right) \rightarrow$ $\mathcal{K}^{0}\left(\widetilde{N}_{B}(\Delta)\right)$ is the restriction map. 
(b) For $|B|=1$, the $K$-dimension of $T^{1}(\Delta)_{-\mathbf{b}}$ is one less than the $K$-dimension of the kernel given in (a).

Rigidity. The simplicial complex $\Delta$ is called $\emptyset$-rigid if $T^{1}(\Delta)_{-\mathbf{b}}=0$ for all $\mathbf{b} \in$ $\{0,1\}^{n}$. For $\emptyset$-rigidity it is enough to check the vanishing of $T^{1}(\Delta)_{-\mathbf{b}}$ for $\mathbf{b} \in\{0,1\}^{n}$ with supp $\mathbf{b} \subseteq[\Delta]$. It follows from Proposition 1.2 that $\Delta$ is rigid if and only if $\operatorname{link}_{\Delta} A$ is $\emptyset$-rigid for all $A \in \Delta$. Thus we will assume $\mathbf{a}=0$ from now on.

As an immediate consequence of Proposition 1.3 one obtains

Corollary 1.4. (a) Suppose that $|B| \geq 2$. Then $T^{1}(\Delta)_{-\mathbf{b}}=\Lambda_{B}(\Delta)$, where

$\Lambda_{B}(\Delta)=\left\{\lambda: N_{B}(\Delta) \rightarrow K:\left.\lambda\right|_{\widetilde{N}_{B}(\Delta)}=0\right.$ and $\lambda(F)=\lambda(G)$ whenever $\left.F \subseteq G\right\}$.

(b) $\operatorname{dim}_{K} T^{1}(\Delta)_{-\mathbf{b}}=\operatorname{dim}_{K} \Lambda_{B}(\Delta)-1$ if $|B|=1$.

Let $B$ be a subset of $[n]$. We define $G_{B}(\Delta)$ to be the graph whose vertex set is $N_{B}(\Delta)$ and for which $\{F, G\}$ is an edge of $G_{B}(\Delta)$ if and only if $F \subsetneq G$ or $G \subsetneq F$. It follows that $\lambda \in \Lambda_{B}(\Delta)$ is constant on the connected components of $G_{B}(\Delta)$. Note that if $|B|=1$, then $\widetilde{N}_{B}(\Delta)=\emptyset$. We see that if $|B| \geq 2$, then

$$
\begin{aligned}
\operatorname{dim}_{K} T^{1}(\Delta)_{-\mathbf{b}}= & \text { number of connected components of } G_{B}(\Delta) \\
& \text { which contain no element of } \widetilde{N}_{B}(\Delta),
\end{aligned}
$$

and if $B=\{i\}$, then

$$
\operatorname{dim}_{K} T^{1}(\Delta)_{-\mathbf{e}_{i}}=\text { number of connected components of } G_{\{i\}}(\Delta)-1
$$

Hence the rigidity of a simplicial complex is independent of the field $K$.

Examples 1.5. (a) Let $2^{[n]}=\langle[n]\rangle$ be the simplex on the vertex set $[n]$. For each $B \subseteq[n]$ we have $\widetilde{N}_{B}\left(2^{[n]}\right)=\emptyset$. This implies that $2^{[n]}$ is $\emptyset$-rigid. Moreover, for each $A \in 2^{[n]}$, its link is a simplex, too. Thus, $2^{[n]}$ is rigid. Of course, this is known before because $K\left[2^{[n]}\right]=K\left[x_{1}, \ldots, x_{n}\right]$.

(b) Fix $n \geq 2$, and let $\Gamma=2^{[n]} \backslash\{[n]\}$ be the boundary of $2^{[n]}$. Then $N_{[n]}(\Gamma)=\{\emptyset\}$, but $\widetilde{N}_{[n]}(\Gamma)=\emptyset$. In particular, $\operatorname{dim}_{K} T^{1}(\Gamma)_{-\mathbf{b}}=1$ for $\mathbf{b}=(1, \ldots, 1)$. Again this follows also directly from the fact that $K[\Gamma]=K\left[x_{1}, \ldots, x_{n}\right] /\left(x_{1} x_{2} \cdots x_{n}\right)$.

(c) let $\Delta=\langle\{1\}, \ldots,\{n\}\rangle$. Then $\Delta$ is 0-dimensional. The set $B=\{1\}$ yields $N_{B}(\Delta)=\{\{2\}, \ldots,\{n\}\}$ and $\widetilde{N}_{B}(\Delta)=\emptyset$. Hence, $T^{1}(\Delta)_{-\mathbf{b}} \neq 0$ for $n \geq 3$, and so $\Delta$ is not rigid. Note that $K[\Delta]=K\left[x_{1}, \ldots, x_{n}\right] /\left(x_{i} x_{j}: i \neq j\right)$.

The ideal $\left(x_{i} x_{j}: i \neq j\right)$ may be interpreted as the edge ideal of the complete graph on the vertex set $[n]$. Rigidity of edge ideals will be discussed in details in Section 3 .

The following lemma tells us when $T^{1}(\Delta)_{-\mathbf{b}}$ vanishes if supp $\mathbf{b} \notin \Delta$. We denote by $2^{B}$ the simplex on the vertex set $B$.

Lemma 1.6. Let $\Delta$ be a simplicial complex on the vertex set $[n]$, and let $\mathbf{b} \in\{0,1\}^{n}$. Let $B=\operatorname{supp} \mathbf{b}$ and assume that $B \notin \Delta$. 
(a) Suppose that $|B| \geq 2$. Then $T^{1}(\Delta)_{-\mathbf{b}}=0$ if and only if $\widetilde{N}_{B}(\Delta) \neq \emptyset$. Moreover, $T^{1}(\Delta)_{-b} \neq 0$ implies that the boundary $2^{B} \backslash\{B\}$ of $2^{B}$ is contained in $\Delta$.

(b) Suppose that $|B|=1$. Then $T^{1}(\Delta)_{-\mathbf{b}}=0$.

Proof. Since $B \notin \Delta$ it follows that $\emptyset \in N_{B}(\Delta)$. Therefore, $\lambda(F)=\lambda(\emptyset)$ for all $\lambda \in \Lambda_{B}(\Delta)$. Thus the $K$-vector space $\Lambda_{B}(\Delta)$ is generated by one element $\lambda_{0}$ which is forced to be the 0-element if $\widetilde{N}_{B}(\Delta) \neq \emptyset$ and which may be chosen to be the constant map with $\lambda_{0}(\emptyset)=1$, otherwise. This proves (a). Also (b) follows from this considerations keeping in mind Corollary 1.4(b). Alternatively, statement (b) follows from Lemma 1.2(a).

Separation. Let, as before, $\Delta$ be a simplicial complex on the vertex set $[n]$. We say that $\Delta$ is separable, if for some $i, I_{\Delta}$ admits a separation for $x_{i}$. Otherwise, we say that $\Delta$ is inseparable. In the further discussions we refer to the conditions (i), (ii) and (iii) for separation, as given in the introduction.

Let $I=I_{\Delta}$ be minimally generated by the monomials $u_{1}, \ldots, u_{m}$. We first observe:

Lemma 1.7. If $J$ is a separation of $I$ for the variable $x_{i}$, then $T^{1}(\Delta)_{-\mathbf{e}_{i}} \neq 0$.

Proof. By condition (iii), $S / I$ is obtained from $S[y] / J$ by reduction modulo a linear form which is a regular element on $S[y] / J$. This implies that $I$ and $J$ are minimally generated by the same number of generators. Let $J$ be minimally generated by $v_{1}, \ldots, v_{m}$. We may assume that $y$ divides $v_{1}, \ldots, v_{k}$ but does not divide the other generators of $J$. We may furthermore assume that for all $i, v_{i}$ is mapped to $u_{i}$ under the $K$-algebra homomorphism (i). Then we may write

$$
J=\left(u_{1}+\left(u_{1} / x_{i}\right)\left(y-x_{i}\right), \ldots, u_{k}+\left(u_{k} / x_{i}\right)\left(y-x_{i}\right), u_{k+1}, \ldots, u_{m}\right) .
$$

From this presentation and by (iii) it follows that $S[y] / J$ is an unobstructed deformation of $S / I$ induced by the element $[\varphi] \in T^{1}(S / I)_{-\mathbf{e}_{i}}$, where $\varphi \in I^{*}$ is the $S$-module homomorphism with $\varphi\left(u_{j}\right)=u_{j} / x_{i}+I$ for $j=1, \ldots, k$ and $\varphi\left(u_{j}\right)=0$, otherwise.

Condition (ii) makes sure that $S[y] / J$ is a non-trivial deformation of $S / I$. Indeed, suppose $[\varphi]=0$. Observe, that $\operatorname{deg} \varphi=-\mathbf{e}_{i}$. Therefore, $\varphi \in\left(\operatorname{Im} \delta^{*}\right)_{-\mathbf{e}_{i}}$, which is the $K$-vector space spanned by $\varphi_{i}=\delta^{*}\left(\partial / \partial x_{i}\right)$. Here $\delta^{*}: \operatorname{Der}_{K}(S) \rightarrow I^{*}$ is the map as defined in the introduction with $\delta^{*}(\partial)(f)=\partial f+I$ for $\partial \in \operatorname{Der}_{K}(S)$ and $f \in I$. It follows that $\varphi=\lambda \varphi_{i}$ for some $\lambda \in K$. Since $\varphi\left(u_{1}\right)=u_{1} / x_{i}+I=\lambda \varphi_{i}\left(u_{1}\right)$ it follows that $\lambda=1$. On the other hand, by condition (ii), there exists $j>k$ such that $x_{i} \mid u_{j}$ and $\varphi\left(u_{j}\right)=I \neq u_{j} / x_{i}+I=\varphi_{i}\left(u_{j}\right)$. This is a contradiction.

It follows from the above result that $\Delta$ is inseparable if $T^{1}(\Delta)_{-\mathbf{e}_{i}}=0$ for $i=$ $1, \ldots, n$. Moreover the deformation $K[y] \rightarrow S[y] / J$ induces an infinitesimal deformation $K[\varepsilon] \rightarrow S[\varepsilon] / \bar{J}$ by reduction modulo $y^{2}$. As explained in the introduction, this infinitesimal deformation yields an element in $T^{1}(\Delta)$. This assignment is called the Kodaira-Spencer map. The arguments given above, even show that the image of the deformation $K[y] \rightarrow S[y] / J$ in $T^{1}(\Delta)$ via the Kodaira-Spencer map is non-trivial. 
Finally we obtain

Theorem 1.8. The following conditions are equivalent:

(a) $\Delta$ is inseparable.

(b) $G_{\{i\}}(\Delta)$ is connected for $i=1, \ldots, n$.

(c) $T^{1}(\Delta)_{-\mathbf{e}_{i}}=0$ for $i=1, \ldots, n$.

Proof. (c) $\Rightarrow$ (a) follows from Lemma 1.7, and (b) $\Longleftrightarrow$ (c) follows from the equality (2) in the preceding subsection.

$(\mathrm{a}) \Rightarrow(\mathrm{b})$ : Suppose $G_{\{i\}}(\Delta)$ is not connected for some $i$. Then the vertex set of $G_{\{i\}}(\Delta)$ can be written as a disjoint union $V\left(G_{\{i\}}(\Delta)\right)=\mathcal{A} \cup \mathcal{B}$ such that for all $F \in \mathcal{A}$ and all $G \in \mathcal{B}$, neither $F \subsetneq G$ nor $G \subsetneq F$.

Let $I=I_{\Delta}, I(\mathcal{A})=\left(x_{F}: F \in \mathcal{A}\right)$ and $I(\mathcal{B})=\left(x_{G}: G \in \mathcal{B}\right)$. Then

$$
I=\left(x_{i} I(\mathcal{A}), x_{i} I(\mathcal{B}), u_{1}, u_{2}, \ldots, u_{t}\right),
$$

where none of the $u_{j}$ is divisible by $x_{i}$.

Note that if $\left\{x_{F_{1}}, \ldots, x_{F_{r}}\right\}$ is the minimal set of monomial generators of $I(\mathcal{A})$ and $\left\{x_{G_{1}}, \ldots, x_{G_{s}}\right\}$ is the minimal set of monomial generators of $I(\mathcal{B})$, then

$$
\left\{x_{i} x_{F_{1}}, \ldots, x_{i} x_{F_{r}}, x_{i} x_{G_{1}}, \ldots, x_{i} x_{G_{s}}\right\}
$$

is the set of monomials of the minimal monomial set of generators of $I$ which are divisible by $x_{i}$. Thus, the ideal $J=\left(y I(\mathcal{A}), x_{i} I(\mathcal{B}), u_{1}, u_{2}, \ldots, u_{t}\right) \subseteq S[y]$ satisfies the conditions (i) and (ii) of a separation of $I$. We will show that $y-x_{i}$ is a non zero-divisor of $S[y] / J$. This will then imply that $\Delta$ is separable, yielding a contradiction.

Indeed, suppose $y-x_{i}$ is a zero-divisor of $S[y] / J$. Then $y-x_{i}$ belongs to a minimal prime ideal $P$ of $J$. Since $P$ is a monomial prime ideal it follows that $y, x_{i} \in P$.

Now let $F \in \mathcal{A}$ and $G \in \mathcal{B}$ and suppose that $F \cup G \in \Delta$. Then $F \cup G \in N_{\{i\}}(\Delta)$, and hence $F \cup G \in \mathcal{A}$ since $F \subsetneq F \cup G$, and similarly $F \cup G \in \mathcal{B}$ since $G \subsetneq F \cup G$. This is a contradiction. Therefore, $F \cup G \notin \Delta$ for all $F \in \mathcal{A}$ and $G \in \mathcal{B}$. This implies that $I(\mathcal{A}) I(\mathcal{B}) \subseteq I$. It follows that $I(\mathcal{A}) I(\mathcal{B}) \subseteq J$, since $x_{i}$ does not divide any of the generators of $I(\mathcal{A}) I(\mathcal{B})$. Now since $I(\mathcal{A}) I(\mathcal{B}) \subseteq P$ we conclude that $I(\mathcal{A}) \subseteq P$ or $I(\mathcal{B}) \subseteq P$. As $P$ is a minimal prime ideal of $J$, we see that $y \notin P$ if $I(\mathcal{A}) \subseteq P$ and $x_{i} \notin P$ if $I(\mathcal{B}) \subseteq P$. In any case we obtain a contradiction.

$k$-Separation. Let $\mathcal{A}$ and $\mathcal{B}$ be two finite collections of sets with $F \cap G=\emptyset$ for all $F \in \mathcal{A}$ and $G \in \mathcal{B}$. As it is common, we denote by $\mathcal{A} * \mathcal{B}$ the join of $\mathcal{A}$ and $\mathcal{B}$, where $\mathcal{A} * \mathcal{B}=\{F \cup G: F \in \mathcal{A}, G \in \mathcal{B}\}$. If $\Gamma$ and $\Sigma$ are simplicial complexes, then the join $\Gamma * \Sigma$ is again a simplicial complex. The vertex set of $\Gamma * \Sigma$ is the set $V(\Gamma) \cup V(\Sigma)$. In [1, Lemma 4.3], it was shown that all $T_{-\mathbf{e}_{i}}^{1}(X)$ vanish whenever $X$ is a combinatorial manifold without boundary. Now we will show that beyond this special case the deformations in degree $-\mathbf{e}_{i}$ are unobstructed. More precisely, we prove the following statement:

Proposition 1.9. Assume that $V(\Delta)=[n]$ and $\operatorname{dim}_{K} T^{1}(\Delta)_{-\mathbf{e}_{i}}=k$. Then there exists a simplicial complex $\widetilde{\Delta}$ on the vertex set $([n] \backslash\{i\}) \cup\left\{v_{0}, \ldots, v_{k}\right\}$ such that $I_{\widetilde{\Delta}}$ is 
obtained from $I_{\Delta}$ by $k$ times separations for the variable $x_{i}$. Moreover $T^{1}(\widetilde{\Delta})_{-\mathbf{b}}=0$ for any $\mathbf{b}$ whose support $\operatorname{supp} \mathbf{b} \subseteq\left\{v_{0}, \ldots, v_{k}\right\}$.

Proof. We may decompose $\Delta$ as a disjoint union

$$
\{\emptyset,\{i\}\} * \operatorname{link}_{\Delta}\{i\} \cup N_{\{i\}}(\Delta) .
$$

Since $\operatorname{dim}_{K} T^{1}(\Delta)_{-\mathbf{e}_{i}}=k$, we have $N_{\{i\}}(\Delta)$ splits into $k+1$ connected components

$$
N_{\{i\}}(\Delta)=A_{0} \cup A_{1} \cup \ldots \cup A_{k} .
$$

As in the proof of Theorem 1.8, we can express $I_{\Delta}$ as

$$
I_{\Delta}=\left(\left\{x_{i} x_{F}: F \in \bigcup_{\ell=0}^{k} A_{\ell}\right\}, u_{1}, \ldots, u_{t}\right)
$$

where the generators $u_{j}$ are not divisible by $x_{i}$.

We define a new simplicial complex $\widetilde{\Delta}$ on the vertex set $([n] \backslash\{i\}) \cup\left\{v_{0}, v_{1}, \ldots, v_{k}\right\}$ by

$$
\widetilde{\Delta}=\Omega * \operatorname{link}_{\Delta}\{i\} \cup \bigcup_{\ell=0}^{k}\left(\Omega_{\ell} * A_{\ell}\right) .
$$

Here $\Omega=\left\langle\left\{v_{0}, \ldots, v_{k}\right\}\right\rangle$, and $\Omega_{\ell}=\left\langle\left\{v_{0}, \ldots, v_{k}\right\} \backslash\left\{v_{\ell}\right\}\right\rangle$ for $\ell=0, \ldots, k$.

Set $T=K\left[x_{1}, \ldots, x_{i-1}, x_{i+1}, \ldots, x_{n}, y_{0}, \ldots, y_{k}\right]$. Then $I_{\widetilde{\Delta}}$ is an ideal of $T$ and

$$
I_{\widetilde{\Delta}}=\left(\left\{y_{\ell} x_{F}: \ell=0, \ldots, k, F \in A_{\ell}\right\}, u_{1}, \ldots, u_{t}\right) .
$$

Theorem 1.7 provides the induction step of proving the $k$-separability. Thus applying induction it can be shown that $S / I$ is isomorphic to $T / I_{\widetilde{\Delta}}$ modulo the regular sequence $y_{1}-y_{0}, \ldots, y_{k}-y_{k-1}$, and furthermore $T^{1}(\widetilde{\Delta})_{-\mathbf{b}}=0$ for any $\mathbf{b}$ whose support supp $\mathbf{b} \subseteq\left\{v_{0}, \ldots, v_{k}\right\}$.

We call $I_{\widetilde{\Delta}}$ a $k$-separation of $I_{\Delta}$.

\section{JoINS, DISJOINT UNIONS AND CIRCS OF SIMPLICIAL COMPLEXES}

In this section we consider simplicial complexes arising from pairs of simplicial complexes and study their behaviour with respect to rigidity. Part of the results will be applied to classify rigid algebras defined by letterplace ideals.

Monomial localization. In the following localization will be one of the tools in the proofs. Let $K$ be a field, $S=K\left[x_{1}, \ldots, x_{n}\right]$ the polynomial ring over $K, I \subseteq S$ a monomial ideal and $P \subseteq S$ a monomial prime ideal. Then $P=P_{F}$ where $F \subseteq[n]$ and $P_{F}=\left(x_{i}: i \in F\right)$.

We observe that if $(S / I)_{P}$ denotes ordinary localization of $S / I$ with respect to the prime ideal $P$, then $(S / I)_{P}=S_{P} / I(P) S_{P}$, where $I(P) \subseteq S(P):=K\left[x_{i}: i \in F\right]$ is the monomial ideal which is obtained from $I$ by the substitution $x_{i} \mapsto 1$ for $i \notin F$. The ideal $I(P)$ is called the monomial localization of $I$ with respect to $P$.

Lemma 2.1. Let $I \subseteq S$ be a monomial ideal, $P \subseteq S$ a monomial prime ideal, and $F \subseteq[n]$. 
(a) $T^{1}(S / I)_{P} \cong\left(T^{1}(S(P) / I(P))\left[x_{i}: i \notin P\right]\right)_{P}$.

(b) Suppose that $T^{1}(S(P) / I(P)) \neq 0$. Then $T^{1}(S / I) \neq 0$.

(c) Let $I$ be a squarefree monomial ideal. Let $\Delta$ be the simplicial complex on the vertex set $[n]$ with $I_{\Delta}=I$. Then $S\left(P_{\bar{F}}\right) / I_{\Delta}\left(P_{\bar{F}}\right)=S\left(P_{\bar{F}}\right) / I_{\Gamma}$, where $\bar{F}=[n] \backslash F$ and $\Gamma=\operatorname{link}_{\Delta}(F)$. In particular, $\Gamma$ is rigid if $\Delta$ is rigid.

Proof. (a) Note that $\operatorname{Der}_{K}(S)=\operatorname{Hom}_{S}\left(\Omega_{S / K}, S\right)$, where $\Omega_{S / K}$ is module of differentials of $S / K$, see [5, Definition, p. 384]. Since $\Omega_{S / K}$ localizes (see [5, Proposition 16.6], the same holds true for $\operatorname{Der}_{K}(S)$. In other words, $\operatorname{Der}_{K}(S)_{P}=\operatorname{Der}_{K}\left(S_{P}\right)$. From this fact one easily deduces that $T^{1}(S / I)$ localizes, that is $T^{1}(S / I)_{P}=T^{1}\left((S / I)_{P}\right)$. Therefore,

$$
\begin{aligned}
T^{1}(S / I)_{P} & \cong T^{1}\left((S / I)_{P}\right) \cong T^{1}\left((S / I(P) S)_{P}\right) \cong T^{1}\left((S(P) / I(P))\left[x_{i}: x_{i} \notin P\right]\right)_{P} \\
& \cong\left(T^{1}(S(P) / I(P))\left[x_{i}: i \notin P\right]\right)_{P} .
\end{aligned}
$$

The last isomorphism follows from the fact that $T^{1}(R[y]) \cong T^{1}(R) \otimes_{R} R[y]=$ $T^{1}(R)[y]$ for a polynomial extension $R \rightarrow R[y]$.

(b) Suppose that $T^{1}(S(P) / I(P)) \neq 0$ and let $\mathfrak{m}_{P}$ be the graded maximal ideal of $S(P)$. Then $T^{1}(S(P) / I(P))_{\mathfrak{m}_{P}} \neq 0$ because $\mathfrak{m}_{P} \in \operatorname{Supp}\left(T^{1}(S(P) / I(P))\right)$. It follows that $\left(T^{1}(S(P) / I(P))\left[x_{i}: i \notin P\right]\right)_{P} \neq 0$, since $\mathfrak{m}_{P} S=P$. Hence the assertion follows from part (a).

(c) Since $\mathcal{F}(\Gamma)=\{G \backslash F: G \in \mathcal{F}(\Delta), F \subseteq G\}$, we obtain that $I_{\Gamma} \subseteq S\left(P_{\bar{F}}\right)$ is given by

$$
I_{\Gamma}=\bigcap_{G \in \mathcal{F}(\Delta), F \subseteq G} P_{\bar{F} \backslash(G \backslash F)}=\bigcap_{G \in \mathcal{F}(\Delta), F \subseteq G} P_{\bar{G}} .
$$

On the other hand,

$$
I_{\Delta}\left(P_{\bar{F}}\right)=\bigcap_{G \in \mathcal{F}(\Delta)} P_{\bar{G}}\left(P_{\bar{F}}\right)=\bigcap_{G \in \mathcal{F}(\Delta), F \subseteq G} P_{\bar{G}} .
$$

Hence $I_{\Gamma}=I_{\Delta}\left(P_{\bar{F}}\right)$.

Note that the fact stated in Lemma 2.1(c) which says that each link of a rigid simplicial complex is again rigid can also be deduced from Proposition 1.2(b).

Example 2.2. Let $I$ be a squarefree monomial ideal of $K\left[x_{1}, \ldots, x_{n}\right]$ generated in degree $(n-1)$.

(a) If $n=3$, then $I$ is rigid if and only if $I$ is generated by two monomials.

(b) If $n \geq 4$, then $I$ is not rigid.

Proof. In the following we may assume that $I$ is not a principal ideal, because in this case $I$ is a complete intersection generated in degree $\geq 2$, and hence $I$ is not rigid.

(a) For $n=3, I$ is an edge ideal of a nonempty simple graph with 3 vertices. Edge ideals will be treated in detail on Section 3. A nonempty simple graph with 3 vertices is an isolated edge or a path of length 2 or a triangle. It follows from Theorem 3.10 that in those graph, only the second is rigid. This proves our result. 
(b) We proceed by induction on $n$. Assume $n=4$. If $I$ is generated by 4 monomials, then the monomial localization $I(P)$ of $I$ with respect to $P=\left(x_{1}, x_{2}, x_{3}\right)$ is the edge ideal of a triangle, and so $I(P)$ is not rigid because of Theorem 3.10. By Lemma 2.1(b) it follows that $I$ is not rigid. If $I$ is generated by 3 monomials, then $I$ is the product of a variable, say $x_{1}$, and the edge ideal $\left(x_{2} x_{3}, x_{2} x_{4}, x_{3} x_{4}\right)$ of a triangle, and so it is not rigid. Indeed, this follows again from Lemma 2.1(b) by monomial localization with respect to $P=\left(x_{2}, x_{3}, x_{4}\right)$. If $I$ is generated by 2 monomials, then it is the product of a monomial of degree 2 and an ideal generated by variables, and again $I$ is not rigid. Assume $n>4$. If $I$ is generated by $n$ monomials, then the monomial localization $I(P)$ of $I$ with respect to $P=\left(x_{1}, x_{2}, x_{3}\right)$ is the edge ideal of a triangle, and so $I$ is not rigid. If $I$ is generated by less than $n$ monomials, then $I$ is the product of a variable and an ideal $J$ which is generated in degree $n-2$ in the remaining $n-1$ variables. It follows from the induction hypothesis that $J$ is not rigid and so $I$ is not rigid.

Joins. Note that the notation "join" has been defined in the first section. Thus, if $\Delta_{1}$ and $\Delta_{2}$ are simplicial complexes on disjoint vertex sets, then the join $\Delta_{1} * \Delta_{2}$ is a simplicial complex on the vertex set $V\left(\Delta_{1}\right) \cup V\left(\Delta_{2}\right)$.

In the remaining part of this subsection we always assume that $\Delta_{1}$ and $\Delta_{2}$ are simplicial complexes with disjoint vertex sets $E_{1}$ and $E_{2}$ respectively.

Proposition 2.3. Let $\mathbf{a}_{i} \in \mathbb{N}^{E_{1} \cup E_{2}}$ and $\mathbf{b}_{i} \in\{0,1\}^{E_{1} \cup E_{2}}$ for $i=1,2$ such that $\operatorname{supp}\left(\mathbf{a}_{i}\right) \cap \operatorname{supp}\left(\mathbf{b}_{i}\right)=\emptyset, \operatorname{supp}\left(\mathbf{a}_{i}\right) \cup \operatorname{supp}\left(\mathbf{b}_{i}\right) \subseteq\left[\Delta_{i}\right]$. Then

$$
T^{1}\left(\Delta_{1} * \Delta_{2}\right) \mathbf{a}_{1}+\mathbf{a}_{2}-\mathbf{b}_{1}-\mathbf{b}_{2}= \begin{cases}0, & \mathbf{b}_{1} \neq 0 \text { and } \mathbf{b}_{2} \neq 0 ; \\ 0, & \text { either } \operatorname{supp}\left(\mathbf{a}_{1}\right) \notin \Delta_{1} \text { or } \operatorname{supp}\left(\mathbf{a}_{2}\right) \notin \Delta_{2} ; \\ T^{1}\left(\Delta_{1}\right)_{\mathbf{a}_{1}-\mathbf{b}_{1},}, & \mathbf{b}_{2}=0 \text { and } \operatorname{supp}\left(\mathbf{a}_{2}\right) \in \Delta_{2} ; \\ T^{1}\left(\Delta_{2}\right)_{\mathbf{a}_{2}-\mathbf{b}_{2}}, & \mathbf{b}_{1}=0 \text { and } \operatorname{supp}\left(\mathbf{a}_{1}\right) \in \Delta_{1} .\end{cases}
$$

In particular $\Delta_{1} * \Delta_{2}$ is rigid if and only if $\Delta_{1}$ and $\Delta_{2}$ are rigid.

Proof. If either $\operatorname{supp}\left(\mathbf{a}_{1}\right) \notin \Delta_{1}$ or $\operatorname{supp}\left(\mathbf{a}_{2}\right) \notin \Delta_{2}$ then $T^{1}\left(\Delta_{1} * \Delta_{2}\right) \mathbf{a}_{1}+\mathbf{a}_{2}-\mathbf{b}_{1}-\mathbf{b}_{2}=0$ by Proposition 1.2. If $\operatorname{supp}\left(\mathbf{a}_{1}\right) \in \Delta_{1}$ and $\operatorname{supp}\left(\mathbf{a}_{2}\right) \in \Delta_{2}$, we will prove the following equality in several steps.

$$
T^{1}\left(\Delta_{1} * \Delta_{2}\right)_{\mathbf{a}}=\left(T^{1}\left(\Delta_{1}\right)\left[y_{1}, \ldots, y_{m}\right]\right)_{\mathbf{a}} \oplus\left(T^{1}\left(\Delta_{2}\right)\left[x_{1}, \ldots, x_{n}\right]\right)_{\mathbf{a}} .
$$

Here, we denote $\mathbf{a}=\mathbf{a}_{1}+\mathbf{a}_{2}-\mathbf{b}_{1}-\mathbf{b}_{2}, E_{1}=\left\{x_{1}, \ldots, x_{n}\right\}$ and $E_{2}=\left\{y_{1}, \ldots, y_{m}\right\}$. Note that Proposition 2.3 follows immediately from this equality.

(i) First, Observing that links commute with joins, it is enough to prove that

$$
T^{1}\left(\Delta_{1} * \Delta_{2}\right)_{-\left(\mathbf{b}_{1}+\mathbf{b}_{2}\right)}=\left(T^{1}\left(\Delta_{1}\right)\left[y_{1}, \ldots, y_{m}\right]\right)_{-\left(\mathbf{b}_{1}+\mathbf{b}_{2}\right)} \oplus\left(T^{1}\left(\Delta_{2}\right)\left[x_{1}, \ldots, x_{n}\right]\right)_{-\left(\mathbf{b}_{1}+\mathbf{b}_{2}\right)},
$$

where $B_{i}:=\operatorname{supp} \mathbf{b}_{i} \subseteq E_{i}$ for $i=1,2$.

(ii) Then we show that

$$
N_{B_{1} \cup B_{2}}\left(\Delta_{1} * \Delta_{2}\right)=\left[N_{B_{1}}\left(\Delta_{1}\right) *\left(\Delta_{2} \backslash B_{2}\right)\right] \cup\left[\left(\Delta_{1} \backslash B_{1}\right) * N_{B_{2}}\left(\Delta_{2}\right)\right],
$$

where $\Delta_{i} \backslash B_{i}=\left\{F \in \Delta_{i}: F \cap B_{i}=\emptyset\right\}$ for $i=1,2$. 
Let $F_{1} \cup F_{2} \in N_{B_{1} \cup B_{2}}\left(\Delta_{1} * \Delta_{2}\right)$ with $F_{i} \in \Delta_{i}$ for $i=1,2$. Then $F_{i} \in \Delta_{i} \backslash B_{i}$ for $i=1,2$ and $\left(F_{1} \cup F_{2}\right) \cup\left(B_{1} \cup B_{2}\right) \notin \Delta_{1} * \Delta_{2}$ by definition. It follows that for at least one $i \in\{1,2\}, F_{i} \cup B_{i} \notin \Delta_{i}$, namely, $F_{i} \in N_{B_{i}}\left(\Delta_{i}\right)$. This actually proves the containment $\subseteq$. The other containment can be proved similarly.

(iii) Suppose that $B_{i} \neq \emptyset$ for $i=1,2$. We will show that both sides of the identity in step (i) vanishes.

Let $F_{1} \cup F_{2} \in N_{B_{1} \cup B_{2}}\left(\Delta_{1} * \Delta_{2}\right)$ with $F_{i} \in \Delta_{i}$ for $i=1,2$. Then $F_{i} \in N_{B_{i}}\left(\Delta_{i}\right)$, for at least one $i \in\{1,2\}$ by the proof of (ii), say $i=1$. It follows that $\left(F_{1} \cup F_{2}\right) \cup$ $B_{1} \notin \Delta_{1} * \Delta_{2}$. Since $B_{1} \subsetneq B_{1} \cup B_{2}$, we have $F_{1} \cup F_{2} \in \widetilde{N}_{B_{1} \cup B_{2}}\left(\Delta_{1} * \Delta_{2}\right)$. Hence $N_{B_{1} \cup B_{2}}\left(\Delta_{1} * \Delta_{2}\right)=\widetilde{N}_{B_{1} \cup B_{2}}\left(\Delta_{1} * \Delta_{2}\right)$. This implies that the left side of the identity in step (i) vanishes.

To see that the right side also vanishes, we note that a multi-homogeneous element in $T^{1}\left(\Delta_{1}\right)\left[y_{1}, \ldots, y_{m}\right]$ has the form $t y_{1}^{a_{1}} \cdots y_{m}^{a_{m}}$, where $t$ is a multi-homogeneous element in $T^{1}\left(\Delta_{1}\right)$ and $a_{i} \geq 0$ for $i=1, \ldots, m$. But $y_{1}^{a_{1}} \cdots y_{m}^{a_{m}}$ has the multihomogeneous degree for which each coordinate is greater or equal than zero. Hence $\left(T^{1}\left(\Delta_{1}\right)\left[y_{1}, \ldots, y_{m}\right]\right)_{-\left(\mathbf{b}_{1}+\mathbf{b}_{2}\right)}=0$. Similarly $\left(T^{1}\left(\Delta_{2}\right)\left[x_{1}, \ldots, x_{n}\right]\right)_{-\left(\mathbf{b}_{1}+\mathbf{b}_{2}\right)}=0$.

(iv) If one of the $B_{i}$ is the empty set, say $B_{2}=\emptyset$, then (ii) implies that

$$
N_{B_{1}}\left(\Delta_{1} * \Delta_{2}\right)=N_{B_{1}}\left(\Delta_{1}\right) * \Delta_{2} \text { and } \tilde{N}_{B_{1}}\left(\Delta_{1} * \Delta_{2}\right)=\tilde{N}_{B_{1}}\left(\Delta_{1}\right) * \Delta_{2} .
$$

This yields $T^{1}\left(\Delta_{1} * \Delta_{2}\right)_{-\mathbf{b}_{1}}=T^{1}\left(\Delta_{1}\right)_{-\mathbf{b}_{1}}=\left(T^{1}\left(\Delta_{1}\right)\left[y_{1}, \ldots, y_{m}\right]\right)_{-\mathbf{b}_{1}}$, as desired.

Proposition 2.3 can be rewritten as the following nice formula, which is suggested by William Bitsch, whose email is william.bitsch@fu-berlin.de.

Corollary 2.4. For all $\mathbf{z} \in \mathbb{Z}^{E_{1} \cup E_{2}}$, we have

$$
T^{1}\left(\Delta_{1} * \Delta_{2}\right)_{\mathbf{z}}=\left(T^{1}\left(\Delta_{1}\right) \otimes k\left[\Delta_{2}\right]\right)_{\mathbf{z}} \oplus\left(T^{1}\left(\Delta_{1}\right) \otimes k\left[\Delta_{2}\right]\right)_{\mathbf{z}} \quad .
$$

Proof. First we write $\mathbf{z}$ as $\mathbf{a}_{1}+\mathbf{a}_{2}-\mathbf{b}_{1}-\mathbf{b}_{2}$ with $\operatorname{supp}\left(\mathbf{a}_{i}\right) \cap \operatorname{supp}\left(\mathbf{b}_{i}\right)=\emptyset, \operatorname{supp}\left(\mathbf{a}_{i}\right) \cup$ $\operatorname{supp}\left(\mathbf{b}_{i}\right) \subseteq E_{i}, \mathbf{a}_{i} \in \mathbb{N}^{E_{1} \cup E_{2}}$ and $\mathbf{b}_{i} \in\{0,1\}^{E_{1} \cup E_{2}}$ for $i=1,2$.

Note that as a multigraded linear $k$-space,

$$
k\left[\Delta_{2}\right] \cong \bigoplus_{\mathbf{a} \in \mathbb{N}^{E_{2}, \operatorname{supp}(\mathbf{a}) \in \Delta_{2}}} k y^{\mathbf{a}} .
$$

Hence

$$
\left(T^{1}\left(\Delta_{1}\right) \otimes k\left[\Delta_{2}\right]\right) \mathbf{a}_{1}+\mathbf{a}_{2}-\mathbf{b}_{1}-\mathbf{b}_{2}=\bigoplus_{\mathbf{a} \in \mathbb{N}^{E_{2}}, \operatorname{supp}(\mathbf{a}) \in \Delta_{2}} T^{1}\left(\Delta_{1}\right)_{\mathbf{a}_{1}+\mathbf{a}_{2}-\mathbf{a}-\mathbf{b}_{1}-\mathbf{b}_{2}}
$$

For $\mathbf{a} \in \mathbb{N}^{E_{2}}$, it is easy to see that $T^{1}\left(\Delta_{1}\right) \mathbf{a}_{1}+\mathbf{a}_{2}-\mathbf{a}-\mathbf{b}_{1}-\mathbf{b}_{2} \neq 0$ if and only $T^{1}\left(\Delta_{1}\right) \mathbf{a}_{1}-\mathbf{b}_{1} \neq$ 0 and $\mathbf{a}=\mathbf{a}_{2}, \mathbf{b}_{2}=0$. This implies

$$
\left(T^{1}\left(\Delta_{1}\right) \otimes k\left[\Delta_{2}\right]\right)_{\mathbf{a}_{1}+\mathbf{a}_{2}-\mathbf{b}_{1}-\mathbf{b}_{2}} \cong \begin{cases}T^{1}\left(\Delta_{1}\right)_{\mathbf{a}_{1}-\mathbf{b}_{1},} & \mathbf{b}_{2}=0 \text { and } \operatorname{supp}\left(\mathbf{a}_{2}\right) \in \Delta_{2} \\ 0, & \text { otherwise }\end{cases}
$$

From this the fomula follows in view of Proposition 2.3 . 
Disjoint unions. Next we consider the simplicial complex $\Delta_{1} \cup \Delta_{2}$ which is the disjoint union of $\Delta_{1}$ and $\Delta_{2}$. The vertex set of $\Delta_{1} \cup \Delta_{2}$ is $V\left(\Delta_{1}\right) \cup V\left(\Delta_{2}\right)$ and $F \in \Delta_{1} \cup \Delta_{2}$ if and only if $F \in \Delta_{1}$ or $F \in \Delta_{2}$.

Theorem 2.5. Let $\Delta_{1} \neq\{\emptyset\}$ and $\Delta_{2} \neq\{\emptyset\}$ be simplicial complexes with disjoint vertex sets. Assume that for $i=1,2, V\left(\Delta_{i}\right)=\left[\Delta_{i}\right]$, that $i s,\{j\} \in \Delta_{i}$ for all $j \in V\left(\Delta_{i}\right)$.

(a) The following conditions are equivalent:

(1) $\Delta_{1} \cup \Delta_{2}$ is inseparable;

(2) $\Delta_{1}$ and $\Delta_{2}$ are simplices.

(b) The following conditions are equivalent:

(1) $\Delta_{1} \cup \Delta_{2}$ is rigid;

(2) $\Delta_{1} \cup \Delta_{2}$ is $\emptyset$-rigid;

(3) $\Delta_{1}$ and $\Delta_{2}$ are simplices with $\operatorname{dim} \Delta_{1}+\operatorname{dim} \Delta_{2}>0$.

Proof. Let $\Delta=\Delta_{1} \cup \Delta_{2}$.

(a) $(1) \Rightarrow(2)$ : Since $\Delta$ is inseparable, Theorem 1.8 implies that the graph $G_{\{i\}}(\Delta)$ is connected for all $i \in V\left(\Delta_{1}\right) \cup V\left(\Delta_{2}\right)$. Let $i \in V\left(\Delta_{1}\right)$. By assumption we have $\{i\} \in \Delta$. It follows that $N_{\{i\}}(\Delta)=N_{\{i\}}\left(\Delta_{1}\right) \cup \Delta_{2} \backslash\{\emptyset\}$. Since $N_{\{i\}}\left(\Delta_{1}\right)$ and $\Delta_{2} \backslash\{\emptyset\}$ belong to different connected components of $G_{\{i\}}(\Delta)$ and since $G_{\{i\}}(\Delta)$ is connected, it follows that either $N_{\{i\}}\left(\Delta_{1}\right)=\emptyset$ or $\Delta_{2} \backslash\{\emptyset\}=\emptyset$. The second case is ruled out by assumption. Hence, $N_{\{i\}}\left(\Delta_{1}\right)=\emptyset$. This implies that $i \in F$ for all $F \in \mathcal{F}\left(\Delta_{1}\right)$. Since $i$ is an arbitrary element in $V\left(\Delta_{1}\right)$ we see that $\mathcal{F}\left(\Delta_{1}\right)=\left\{V\left(\Delta_{1}\right)\right\}$. Starting with $i \in V\left(\Delta_{2}\right)$, the same argument proves that $\Delta_{2}$ is also a simplex.

$(2) \Rightarrow(1)$ : By Theorem 1.8, it is enough to show that $G_{\{i\}}(\Delta)$ is connected for all $i \in V\left(\Delta_{1}\right) \cup V\left(\Delta_{2}\right)$. Let $i \in V\left(\Delta_{1}\right)$. As mentioned above we have $N_{\{i\}}(\Delta)=$ $N_{\{i\}}\left(\Delta_{1}\right) \cup \Delta_{2} \backslash\{\emptyset\}$. Since $\Delta_{1}$ is a simplex and $\{i\} \in \Delta_{1}$ it follows that $N_{\{i\}}\left(\Delta_{1}\right)=\emptyset$. Thus $N_{\{i\}}(\Delta)=\Delta_{2} \backslash\{\emptyset\}$. Therefore $G_{\{i\}}(\Delta)$ is connected because $\Delta_{2}$ is a simplex. A similar argument shows that $G_{\{i\}}(\Delta)$ is also connected for all $i \in V\left(\Delta_{2}\right)$.

(b) $(1) \Rightarrow(2)$ is obvious.

$(2) \Rightarrow(3)$ : Since $\Delta$ is $\emptyset$-rigid we have $T^{1}(\Delta)_{-\mathbf{b}}=0$ for all $\mathbf{b} \in\{0,1\}^{V\left(\Delta_{1}\right) \cup V\left(\Delta_{2}\right)}$. In particular, $T^{1}(\Delta)_{-\mathbf{e}_{i}}=0$ for all $i \in V\left(\Delta_{1}\right) \cup V\left(\Delta_{2}\right)$. So by Theorem 1.8, $\Delta$ is inseparable. Thus using part (a) we have that $\Delta_{1}$ and $\Delta_{2}$ are simplices. Suppose that $\operatorname{dim} \Delta_{1}+\operatorname{dim} \Delta_{2}=0$. Then $\operatorname{dim} \Delta_{1}=\operatorname{dim} \Delta_{2}=0$, and hence $I_{\Delta}$ is of the form $(x y)$. It follows that $T^{1}(\Delta)_{-(1,1)} \neq 0$, a contradiction.

$(3) \Rightarrow(1)$ : The assumptions imply that $I_{\Delta}$ is of the form $\left(x_{i} y_{j}: i=1, \ldots, n, j=\right.$ $1, \ldots, m)$ with $n \geq 2$ or $m \geq 2$. Corollary 2.11 implies that $\Delta$ is rigid.

Corollary 2.6. Let $\Delta$ be simplicial complex. Then $\Delta$ is separable if $\Delta$ has more than two connected components.

Circs. For $i=1,2$, let $\Delta_{i}$ be a simplicial complex on the vertex set $V_{i}$ and assume that $V_{1} \cap V_{2}=\emptyset$. Then the circ of $\Delta_{1}$ and $\Delta_{2}$ is the simplicial complex $\Delta_{1} \circ \Delta_{2}$ with vertex set $V_{1} \cup V_{2}$ whose faces are those subsets $F$ of $V_{1} \cup V_{2}$ for which either $F \cap V_{1}$ is a face of $\Delta_{1}$ or $F \cap V_{2}$ is a face of $\Delta_{2}$. 
It is worthwhile to note that if $I_{\Delta_{1}} \subseteq S_{1}=K\left[x_{1}, \ldots, x_{n}\right]$ and $I_{\Delta_{2}} \subseteq S_{2}=$ $K\left[y_{1}, \ldots, y_{m}\right]$ then $I_{\Delta_{1} \circ \Delta_{2}}=I_{\Delta_{1}} I_{\Delta_{2}} S$, where $S=K\left[x_{1}, \ldots, x_{n}, y_{1} \ldots, y_{m}\right]$.

In the following we set $M_{B}(\Delta)=\{F \subseteq V(\Delta): F \notin \Delta$ and $F \cap B=\emptyset\}$ for any $B \subseteq V(\Delta)$. For later use we list a few obvious facts in the next lemmata.

Lemma 2.7. Let $B=B_{1} \cup B_{2}$ with $\emptyset \neq B_{i} \subseteq V_{i}$ for $i=1$,2. Then

(a) $N_{B}\left(\Delta_{1} \circ \Delta_{2}\right)=N_{B_{1}}\left(\Delta_{1}\right) * N_{B_{2}}\left(\Delta_{2}\right) \cup N_{B_{1}}\left(\Delta_{1}\right) * M_{B_{2}}\left(\Delta_{2}\right)$ $\cup M_{B_{1}}\left(\Delta_{1}\right) * N_{B_{2}}\left(\Delta_{2}\right)$.

(b) $N_{B_{1}}\left(\Delta_{1} \circ \Delta_{2}\right)=N_{B_{1}}\left(\Delta_{1}\right) * M_{\emptyset}\left(\Delta_{2}\right)$ and $N_{B_{2}}\left(\Delta_{1} \circ \Delta_{2}\right)=M_{\emptyset}\left(\Delta_{1}\right) * N_{B_{2}}\left(\Delta_{2}\right)$.

Lemma 2.8. Let $B=B_{1} \cup B_{2}$ with $\emptyset \neq B_{i} \subseteq V_{i}$ for $i=1,2$. Then

(a) $\widetilde{N}_{B}\left(\Delta_{1} \circ \Delta_{2}\right)=N_{B_{1}}\left(\Delta_{1}\right) * \widetilde{N}_{B_{2}}\left(\Delta_{2}\right) \cup \widetilde{N}_{B_{1}}\left(\Delta_{1}\right) * N_{B_{2}}\left(\Delta_{2}\right)$ $\cup N_{B_{1}}\left(\Delta_{1}\right) * M_{B_{2}}\left(\Delta_{2}\right) \cup M_{B_{1}}\left(\Delta_{1}\right) * N_{B_{2}}\left(\Delta_{2}\right)$.

(b) $\widetilde{N}_{B_{1}}\left(\Delta_{1} \circ \Delta_{2}\right)=\widetilde{N}_{B_{1}}\left(\Delta_{1}\right) * M_{\emptyset}\left(\Delta_{2}\right)$ and $\widetilde{N}_{B_{2}}\left(\Delta_{1} \circ \Delta_{2}\right)=M_{\emptyset}\left(\Delta_{1}\right) * \widetilde{N}_{B_{2}}\left(\Delta_{2}\right)$.

We use these lemmata to prove

Proposition 2.9. Let $\Delta_{1}$ and $\Delta_{2}$ be two simplicial complexes on the vertex sets $V_{1}$ and $V_{2}$, respectively, and let $\mathbf{b}_{i} \in\{0,1\}^{V_{i}}, B_{i}=\operatorname{supp} \mathbf{b}_{i}$ for $i=1,2$. Assume that $I_{\Delta_{i}} \neq 0$ for $i=1,2$.

(a) Suppose that $\mathbf{b}_{1}, \mathbf{b}_{2} \neq 0$. Then

$T^{1}\left(\Delta_{1} \circ \Delta_{2}\right)_{-\left(\mathbf{b}_{1}+\mathbf{b}_{2}\right)} \cong \begin{cases}K, & \text { if } \widetilde{N}_{B_{1} \cup B_{2}}\left(\Delta_{1} \circ \Delta_{2}\right)=\emptyset \text { and } N_{B_{1} \cup B_{2}}\left(\Delta_{1} \circ \Delta_{2}\right) \neq \emptyset, \\ 0, & \text { otherwise. }\end{cases}$

(b) $\operatorname{dim}_{K} T^{1}\left(\Delta_{1} \circ \Delta_{2}\right)_{-\mathbf{b}_{i}}=\operatorname{dim}_{K} T^{1}\left(\Delta_{i}\right)_{-\mathbf{b}_{i}}$ for $i=1,2$.

Proof. First we observe that for each $i=1,2, I_{\Delta_{i}} \neq 0$ if and only if $V_{i} \notin \Delta_{i}$, which is equivalent to $M_{\phi}\left(\Delta_{i}\right) \neq \emptyset$.

(a) Assume that $\widetilde{N}_{B_{1} \cup B_{2}}\left(\Delta_{1} \circ \Delta_{2}\right)=\emptyset$ and $N_{B_{1} \cup B_{2}}\left(\Delta_{1} \circ \Delta_{2}\right) \neq \emptyset$. Lemma2.7(a) together with Lemma 2.8(a) imply that $N_{B_{1}}\left(\Delta_{1}\right) * N_{B_{2}}\left(\Delta_{2}\right) \neq \emptyset$. Therefore $N_{B_{i}}\left(\Delta_{i}\right) \neq$ $\emptyset$ for $i=1,2$. Since $\widetilde{N}_{B_{1} \cup B_{2}}\left(\Delta_{1} \circ \Delta_{2}\right)=\emptyset$ it follows from Lemma 2.8(a) that $N_{B_{1}}\left(\Delta_{1}\right) * \widetilde{N}_{B_{2}}\left(\Delta_{2}\right)=\emptyset$ and $\widetilde{N}_{B_{1}}\left(\Delta_{1}\right) * N_{B_{2}}\left(\Delta_{2}\right)=\emptyset$, and so $\widetilde{N}_{B_{i}}\left(\Delta_{i}\right)=\emptyset$ for $i=1,2$. The same argument shows that $M_{B_{i}}\left(\Delta_{i}\right)=\emptyset$ for $i=1,2$.

Since $M_{B_{i}}\left(\Delta_{i}\right)=\emptyset$ for $i=1,2$, we have $V_{i} \backslash B_{i} \in \Delta_{i}$ and hence $V_{i} \backslash B_{i} \in N_{B_{i}}\left(\Delta_{i}\right)$ for $i=1$, 2. It follows that any $\lambda \in T^{1}\left(\Delta_{1} \circ \Delta_{2}\right)_{-\left(\mathbf{b}_{1}+\mathbf{b}_{2}\right)}$ is constant on $N_{B_{1} \cup B_{2}}\left(\Delta_{1} \circ \Delta_{2}\right)$, because for any $F_{1} \cup F_{2} \in N_{B_{1} \cup B_{2}}\left(\Delta_{1} \circ \Delta_{2}\right)$ with $F_{i} \subseteq V_{i}$ for $i=1,2$ we have $F_{1} \cup F_{2} \subseteq\left(V_{1} \backslash B_{1}\right) \cup\left(V_{2} \backslash B_{2}\right)$. Hence $T^{1}\left(\Delta_{1} \circ \Delta_{2}\right)_{-\left(\mathbf{b}_{1}+\mathbf{b}_{2}\right)} \cong K$.

If $N_{B_{1} \cup B_{2}}\left(\Delta_{1} \circ \Delta_{2}\right)=\emptyset$, then $T^{1}\left(\Delta_{1} \circ \Delta_{2}\right)_{-\left(\mathbf{b}_{1}+\mathbf{b}_{2}\right)}=0$. Assume now that $\widetilde{N}_{B_{1} \cup B_{2}}\left(\Delta_{1} \circ \Delta_{2}\right) \neq \emptyset$. Let $\lambda \in \Lambda^{1}\left(\Delta_{1} \circ \Delta_{2}\right)_{-\left(\mathbf{b}_{1}+\mathbf{b}_{2}\right)}$ (see its definition in Corollary (1.4) and $F_{1} \cup F_{2} \in N_{B_{1} \cup B_{2}}\left(\Delta_{1} \circ \Delta_{2}\right) \backslash \widetilde{N}_{B_{1} \cup B_{2}}\left(\Delta_{1} \circ \Delta_{2}\right)$ with $F_{i} \subseteq V_{i}, i=1$, 2. It follows from Lemmata 2.7(a) and 2.8(a) that $F_{i} \in N_{B_{i}}\left(\Delta_{i}\right) \backslash \widetilde{N}_{B_{i}}\left(\Delta_{i}\right)$ for $i=1,2$. By Lemma 2.8(a), at least one of $\widetilde{N}_{B_{i}}\left(\Delta_{i}\right), i=1,2$ and $M_{B_{i}}\left(\Delta_{i}\right), i=1,2$ is nonempty. 
First suppose that $M_{B_{1}}\left(\Delta_{1}\right) \neq \emptyset$. Then we take $G_{1} \in M_{B_{1}}\left(\Delta_{1}\right)$. Note that $G_{1} \cup F_{2} \in \tilde{N}_{B_{1} \cup B_{2}}\left(\Delta_{1} \circ \Delta_{2}\right)$ and $\left(G_{1} \cup F_{2}\right) \cup\left(F_{1} \cup F_{2}\right) \in \Delta_{1} \circ \Delta_{2}$. Therefore $\lambda\left(F_{1} \cup F_{2}\right)=0$. Similarly, we can conclude that $\lambda\left(F_{1} \cup F_{2}\right)=0$ if $M_{B_{2}}\left(\Delta_{2}\right) \neq \emptyset$ or $\widetilde{N}_{B_{i}}\left(\Delta_{i}\right) \neq \emptyset$ for $i=1$ or $i=2$. So we have proved that $\lambda=0$ in any case. Thus $T^{1}\left(\Delta_{1} \circ \Delta_{2}\right)_{-\left(\mathbf{b}_{1}+\mathbf{b}_{2}\right)}=0$.

(b) We prove the statement for $i=1$. The same argument holds for $i=2$. Since $M_{\emptyset}\left(\Delta_{2}\right) \neq \emptyset$, we have $N_{B_{1} \cup B_{2}}\left(\Delta_{1} \circ \Delta_{2}\right)=\emptyset$ if and only if $N_{B_{1}}\left(\Delta_{1}\right)=\emptyset$ by Lemma 2.7(b). Hence we only need to consider the case when $N_{B_{1} \cup B_{2}}\left(\Delta_{1} \circ \Delta_{2}\right) \neq \emptyset$.

We define a homomorphism $\varphi$ from $\Lambda^{1}\left(\Delta_{1}\right)_{-\mathbf{b}_{1}}$ to $\Lambda^{1}\left(\Delta_{1} \circ \Delta_{2}\right)_{-\mathbf{b}_{1}}$ as follows: for $\lambda_{1} \in \Lambda^{1}\left(\Delta_{1}\right)_{-\mathbf{b}_{1}}, \varphi\left(\lambda_{1}\right)$ is given by $\varphi\left(\lambda_{1}\right)\left(F_{1} \cup G_{1}\right)=\lambda_{1}\left(F_{1}\right)$ for any $F_{1} \in$ $N_{B_{1}}\left(\Delta_{1}\right)$ and $G_{1} \in M_{\emptyset}\left(\Delta_{2}\right)$. One can check that $\varphi\left(\lambda_{1}\right)$ belongs to $\Lambda^{1}\left(\Delta_{1} \circ \Delta_{2}\right)_{-\mathbf{b}_{1}}$ and that $\varphi$ is injective. To see that $\varphi$ is surjective, one only need to notice that for any $\lambda \in \Lambda^{1}\left(\Delta_{1} \circ \Delta_{2}\right)_{-\mathbf{b}_{1}}, \lambda\left(F_{1} \cup G_{1}\right)=\lambda\left(F_{1} \cup G_{2}\right)$ for any $F_{1} \in N_{B_{1}}\left(\Delta_{1}\right)$ and $G_{1}, G_{2} \in M_{\emptyset}\left(\Delta_{2}\right)$. Hence $\varphi$ is an isomorphism of $K$-vector spaces. This implies that $\operatorname{dim}_{K} T^{1}\left(\Delta_{1} \circ \Delta_{2}\right)_{-\mathbf{b}_{1}}=\operatorname{dim}_{K} T^{1}\left(\Delta_{1}\right)_{-\mathbf{b}_{1}}$, using Corollary 1.4 .

For simplicity we say that a simplicial complex $\Delta$ on the vertex set $[n]$ is special if either $\Delta$ has a unique facet, which is a maximal proper subset of $[n]$, or $\Delta$ has exactly two facets, one of which contains a unique element say $i$ and the other one of which is $[n] \backslash\{i\}$. We see that $\Delta$ is special if and only if $I_{\Delta}$ is of the form $z P$, where $z$ is a variable in $S$ with $z \notin P$, and $P$ is either a monomial prime ideal of $S$ or $P=S$.

Theorem 2.10. (a) Suppose that $\Delta_{1} \circ \Delta_{2}$ is rigid, and that $I_{\Delta_{i}} \neq 0$ for $i=1,2$. Then $\Delta_{1}$ and $\Delta_{2}$ are rigid.

(b) Conversely, suppose that $\Delta_{1}$ and $\Delta_{2}$ are rigid simplicial complexes with disjoint vertex sets $V_{1}$ and $V_{2}$, respectively. Then $\Delta_{1} \circ \Delta_{2}$ is rigid if and only if for either $j=1$ or $j=2, \operatorname{link}_{\Delta_{j}} F$ is not special for all $F \in \Delta_{j}$. In algebraic terms, for $j=1$ or $j=2$, none of the monomial localizations of $I_{\Delta_{j}}$ is of the form $z P$, where $z$ is a variable in $S_{j}$ with $z \notin P$, and $P \subseteq S_{j}$ is either a monomial prime ideal or $P=S_{j}$.

Proof. Let $S_{1}=K\left[x_{1}, \ldots, x_{n}\right], S_{2}=K\left[y_{1}, \ldots, y_{m}\right]$ and $S=K\left[x_{1}, \ldots, x_{n}, y_{1}, \ldots, y_{n}\right]$. We may assume that $I_{\Delta_{1}} \subseteq S_{1}$ and $I_{\Delta_{2}} \subseteq S_{2}$.

(a) Let $P_{1}=\left(x_{1}, \ldots, x_{n}\right)$ and $P_{2}=\left(y_{1}, \ldots, y_{m}\right)$. Since

$$
I_{\Delta_{1} \circ \Delta_{2}}\left(P_{i}\right)=\left(I_{\Delta_{1}} I_{\Delta_{2}} S\right)\left(P_{i}\right)=I_{\Delta_{i}}
$$

for $i=1,2$, we see that

$$
T^{1}\left(K\left[\Delta_{1} \circ \Delta_{2}\right]\left(P_{i}\right)\right) \cong T^{1}\left(K\left[\Delta_{i}\right]\right) .
$$

Thus the assertion follows from Lemma 2.1(b).

(b) First suppose that $\Delta_{1} \circ \Delta_{2}$ is rigid, and suppose that there exist $F_{1} \in \Delta_{1}$ and $F_{2} \in \Delta_{2}$ such that the Stanley-Reisner ideal $I_{\text {link }_{\Delta_{j}} F_{j}}$ is of the form $z_{j} P_{j}$ where $z_{j}$ is a variable in $S_{j}$ with $z_{j} \notin P_{j}, P_{j} \subseteq S_{j}$ is either a monomial prime ideal or $P_{j}=S_{j}$. Since $\operatorname{link}_{\Delta_{1} \circ \Delta_{2}} F_{1} \cup F_{2}=\operatorname{link}_{\Delta_{1}} F_{1} \circ \operatorname{link}_{\Delta_{2}} F_{2}$, it follows that

$$
\left(I_{\text {link }_{\Delta_{1} \circ \Delta_{2}} F_{1} \cup F_{2}}\right) S=I_{\text {link }_{\Delta_{1}} F_{1}} I_{\text {link }_{\Delta_{2}} F_{2}} S=\left(z_{1} z_{2}\right) P_{1} P_{2} S
$$


is not rigid because the ideal $\left(z_{1} z_{2}\right)$ is not rigid, a contradiction (see Proposition 1.2(b) or Lemma 2.1(c)).

Suppose that $\Delta_{1} \circ \Delta_{2}$ is not rigid. Then there exist $\mathbf{a}, \mathbf{b} \in\{0,1\}^{V_{1} \cup V_{2}}$ with supp $\mathbf{a} \cap \operatorname{supp} \mathbf{b}=\emptyset$ such that $T^{1}\left(\Delta_{1} \circ \Delta_{2}\right) \mathbf{a - b} \neq 0$. Let $\mathbf{a}=\mathbf{a}_{1}+\mathbf{a}_{2}$ and $\mathbf{b}=\mathbf{b}_{1}+\mathbf{b}_{2}$ with $\mathbf{a}_{1}, \mathbf{b}_{1} \in\{0,1\}^{V_{1}}$ and $\mathbf{a}_{2}, \mathbf{b}_{2} \in\{0,1\}^{V_{2}}$. We set $B=\operatorname{supp} \mathbf{b}, B_{i}=\operatorname{supp} \mathbf{b}_{i}$ for $i=1,2$, and denote by $\Gamma_{i}$ the simplicial complex $\operatorname{link}_{\Delta_{i}}\left(\operatorname{supp} \mathbf{a}_{i}\right)$ for $i=1,2$. Since $\operatorname{link}_{\Delta_{1} \circ \Delta_{2}}(\operatorname{supp} \mathbf{a})=\Gamma_{1} \circ \Gamma_{2}$, we have $T^{1}\left(\Gamma_{1} \circ \Gamma_{2}\right)_{-\mathbf{b}} \neq 0$ by using Proposition [1.2(b). Note that $\Gamma_{i}$ is rigid for $i=1,2$ by Lemma 2.1(c).

Since $T^{1}\left(\Gamma_{1} \circ \Gamma_{2}\right)_{-\mathbf{b}} \neq 0$ we have $\left(I_{\Gamma_{1}} I_{\Gamma_{2}}\right)=I_{\Gamma_{1} \circ \Gamma_{2}} \neq 0$. This implies that $I_{\Gamma_{i}} \neq 0$ for $i=1,2$. Suppose $\mathbf{b}_{1}=0$. Since $T^{1}\left(\Gamma_{1} \circ \Gamma_{2}\right)_{-\mathbf{b}_{2}}=T^{1}\left(\Gamma_{2}\right)_{-\mathbf{b}_{2}}$ (see Proposition $2.9(\mathrm{~b}))$, we have $T^{1}\left(\Gamma_{1} \circ \Gamma_{2}\right)_{-\mathbf{b}_{2}}=0$, because $\Gamma_{2}$ is rigid. This is a contradiction. Therefore $\mathbf{b}_{1} \neq 0$, and similarly $\mathbf{b}_{2} \neq 0$. Now Proposition 2.9)(a) implies that $N_{B_{1} \cup B_{2}}\left(\Gamma_{1} \circ \Gamma_{2}\right) \neq \emptyset$ and $\widetilde{N}_{B_{1} \cup B_{2}}\left(\Gamma_{1} \circ \Gamma_{2}\right)=\emptyset$. By a similar argument used in Proposition 2.9, we have $N_{B_{i}}\left(\Gamma_{i}\right) \neq \emptyset$ and $M_{B_{i}}\left(\Gamma_{i}\right)=\widetilde{N}_{B_{i}}\left(\Gamma_{i}\right)=\emptyset$ for $i=1,2$. Therefore, for $i=1,2$ the map $\lambda_{i}: N_{B_{i}}\left(\Gamma_{i}\right) \rightarrow K$, which maps each element of $N_{B_{i}}\left(\Gamma_{i}\right)$ to 1 , defines a nonzero element in $\Lambda\left(\Gamma_{i}\right)_{-\mathbf{b}_{i}}$. Since $\Gamma_{i}$ is rigid it follows from Corollary 1.4(a) that $\left|B_{i}\right|=1$ for $i=1,2$. Without loss of generality we may assume that $B_{1}=\left\{x_{1}\right\}$ and $B_{2}=\left\{y_{1}\right\}$. Then $V\left(\Gamma_{1}\right) \backslash\left\{x_{1}\right\} \in \Gamma_{1}$ and $V\left(\Gamma_{2}\right) \backslash\left\{y_{1}\right\} \in \Gamma_{2}$ since $M_{B_{i}}\left(\Gamma_{i}\right)=\emptyset$ for $i=1,2$. Now we consider the following cases:

Case 1: $\left|V\left(\Gamma_{1}\right)\right|=1$. Since $I_{\Gamma_{1}}$ is not zero we have $I_{\Gamma_{1}}=\left(x_{1}\right)$ and so $\Gamma_{1}$ is special. Case 2: $\left|V\left(\Gamma_{1}\right)\right|=2$. Assume $V\left(\Gamma_{1}\right)=\left\{x_{1}, x_{2}\right\}$. Then $\left\{x_{2}\right\} \in \Gamma_{1}$. Since $I_{\Gamma_{1}} \neq 0$, we have $\left\{x_{1}, x_{2}\right\} \notin \Gamma_{1}$ and since $I_{\Gamma_{1}}$ is rigid we have $\Gamma_{1} \neq\left\langle\left\{x_{1}\right\},\left\{x_{2}\right\}\right\rangle$. Hence $\Gamma_{1}=\left\langle\left\{x_{2}\right\}\right\rangle$ and $I_{\Gamma_{1}}=\left(x_{1}\right)$. This implies that $\Gamma_{1}$ is special again.

Case 3: $\left|V\left(\Gamma_{1}\right)\right| \geq 3$. Since $V\left(\Gamma_{1}\right) \backslash\left\{x_{1}\right\} \in \Gamma_{1}$, we have either $\Gamma_{1}=\left\langle V\left(\Gamma_{1}\right) \backslash\left\{x_{1}\right\}\right\rangle$ or $\Gamma_{1}=\left\langle V\left(\Gamma_{1}\right) \backslash\left\{x_{1}\right\}, F_{1}, \ldots, F_{k}\right\rangle$, where $k \geq 1$ and $x_{1} \in F_{j}$ for $j=1, \ldots, k$. In the first case we have $I_{\Gamma_{1}}=\left(x_{1}\right)$ and we are done. In the second one, we set $G=F_{1} \backslash\left\{x_{1}\right\}$. Then $\operatorname{link}_{\Gamma_{1}} G=\left\langle V\left(\Gamma_{1}\right) \backslash F_{1},\left\{x_{1}\right\}\right\rangle$ and $V\left(\operatorname{link}_{\Gamma_{1}} G\right)=\left(V\left(\Gamma_{1}\right) \backslash F_{1}\right) \cup\left\{x_{1}\right\}$. Write $V\left(\Gamma_{1}\right) \backslash F_{1}=\left\{x_{2}, \ldots, x_{s}\right\}$. Since $\operatorname{link}_{\Gamma_{1}} G$ is rigid, we have $s \geq 3$ and so $I_{\operatorname{link}_{\Gamma_{1}} G}=$ $\left(x_{1}\right)\left(x_{2}, \ldots, x_{s}\right)=x_{1} P$. This also implies that $\Gamma_{1}$ is special.

The similar argument is applied to $\Gamma_{2}$.

Corollary 2.11. (a) Let $I$ be a squarefree monomial ideal and let $P$ be a nonprincipal monomial prime ideal in a disjoint set of variables. Then IP is rigid if and only if $I$ is rigid.

(b) Let $P_{1}, \ldots, P_{t}$ be monomial prime ideals generated by pairwise disjoint sets of variables. Then $\prod_{i=1}^{t} P_{i}$ is rigid if and only if at most one $P_{i}$ is a principal ideal.

Proof. (a) Let $\Delta_{1}$ and $\Delta_{2}$ be two simplicial complexes with $I_{\Delta_{1}}=I$ and $I_{\Delta_{2}}=P$. Suppose $I P$ is rigid. It follows from Theorem 2.10(a) that $I$ is rigid. Conversely, suppose that $I$ is rigid. Note that the links of $\Delta_{2}$ correspond to monomial localizations of $P$ by Lemma 2.1(c). Since monomial localization of $P$ with respect to any monomial prime ideal is never of the form $(z)$ or $z Q$ with $Q$ a monomial prime ideal, Theorem 2.10(b) yields the desired conclusion.

(b) follows immediately from (a). 
Letterplace ideals. We conclude this section with applications to letterplace ideals. In [4], letterplace and co-letterplace ideals are introduced and it is shown that these are all inseparable monomial ideals. In this section we consider rigidity of this class of ideals.

More generally, let $\mathcal{P}$ and $\mathcal{Q}$ be two partially ordered sets. A map $\varphi: \mathcal{P} \rightarrow \mathcal{Q}$ is called isotone or order preserving, if $p \leq p^{\prime}$ implies $\varphi(p) \leq \varphi\left(p^{\prime}\right)$. The set of isotone maps is denoted by $\operatorname{Hom}(\mathcal{P}, \mathcal{Q})$. Note that $\operatorname{Hom}(\mathcal{P}, \mathcal{Q})$ is again a partially ordered set with $\phi \leq \psi$ if $\phi(p) \leq \psi(p)$ for all $p \in \mathcal{P}$.

We fix a field $K$ and consider the polynomial ring $S$ over $K$ in the variables $x_{p, q}$ with $p \in \mathcal{P}$ and $q \in \mathcal{Q}$. Attached to $\mathcal{P}$ and $\mathcal{Q}$ we define the monomial ideal $L(\mathcal{P}, \mathcal{Q}) \subseteq S$ generated by the monomials

$$
u_{\varphi}=\prod_{p \in \mathcal{P}} x_{p, \varphi(p)}, \quad \varphi \in \operatorname{Hom}(\mathcal{P}, \mathcal{Q}) .
$$

Theorem 2.12. Let $\mathcal{P}$ and $\mathcal{Q}$ be finite posets. The following conditions are equivalent:

(a) $L(\mathcal{P}, \mathcal{Q})$ is rigid.

(b) No two distinct elements of $\mathcal{P}$ are comparable.

Proof. (a) $\Rightarrow(\mathrm{b})$ : Assume there exist $a, b \in \mathcal{P}$ with $a<b$. We consider the monomial prime ideal $P=\left(x_{p, q}: p \in\{a, b\}, q \in \mathcal{Q}\right)$, and claim that $L(\mathcal{P}, \mathcal{Q})(P)=$ $L(\{a, b\}, \mathcal{Q})$, where $\{a, b\}$ is the poset with $a<b$. In fact, for any minimal generator $u \in L(\mathcal{P}, \mathcal{Q})(P)$, there exists $\varphi \in \operatorname{Hom}(\mathcal{P}, \mathcal{Q})$ such that $u$ is obtained from $u_{\varphi}$ by setting $x_{p, \varphi(p)}=1$ if $p \notin\{a, b\}$, that is, $u=x_{a, \varphi(a)} x_{b, \varphi(b)}$. This proves $L(\mathcal{P}, \mathcal{Q})(P) \subseteq$ $L(\{a, b\}, \mathcal{Q})$.

Conversely, let $u=x_{a, c} x_{b, d} \in L(\{a, b\}, \mathcal{Q})$, where $c, d \in \mathcal{Q}$ and $c \leq d$. Let $n=|\mathcal{P}|$. Since any finite partial order can be extended to a total order, there exists an isotone bijective map from $\mathcal{P}$ to $[n]$, which we denote by $f$. We now define a map $\varphi: \mathcal{P} \rightarrow \mathcal{Q}$ as follows:

$$
\varphi(p)= \begin{cases}c, & \text { if } f(p)<f(b), \\ d, & \text { otherwise. }\end{cases}
$$

Then $\varphi \in \operatorname{Hom}(\mathcal{P}, \mathcal{Q})$ and $\varphi(a)=c, \varphi(b)=d$, and hence $u \in L(\mathcal{P}, \mathcal{Q})(P)$. Thus our claim follows.

It follows from Corollary 3.6 and its proof that $S(P) / L(\{a, b\}, \mathcal{Q})$ is not rigid. Therefore, Lemma 2.1(b) implies that $S / L(\mathcal{P}, \mathcal{Q})$ is not rigid.

(b) $\Rightarrow\left(\right.$ a): Let $\mathcal{P}=\left\{p_{1}, \ldots, p_{m}\right\}$ with $p_{i}$ and $p_{j}$ incomparable for all $i \neq j$ and let $\mathcal{Q}=\left\{q_{1}, \ldots, q_{n}\right\}$. Then

$$
L(\mathcal{P}, \mathcal{Q})=\prod_{i=1}^{m}\left(x_{p_{i}, q_{1}}, x_{p_{i}, q_{2}}, \ldots, x_{p_{i}, q_{n}}\right) .
$$

Thus the assertion follows from Corollary 2.11(b).

For an integer $n \in \mathbb{N}$ we denote by $[n]$ the totally ordered set $\{1<2<\cdots<n\}$. The ideal $L([n], \mathcal{P})$ is called the $n$th letterplace ideal, while $L(\mathcal{P},[n])$ is called the $n t h$ co-letterplace ideal. They are Alexander dual to each other if we identify $x_{i, p}$ with 
$x_{p, i}$ for any $i \in[n]$ and $p \in \mathcal{P}$. In particular, the facets of the simplicial complex associated with $L([n], \mathcal{P})$ are in bijection with the generators of $L(\mathcal{P},[n])$, and vice versa.

Corollary 2.13. Let $\mathcal{P}$ a finite poset.

(a) $L([n], \mathcal{P})$ is rigid if and only if $n=1$.

(b) $L(\mathcal{P},[n])$ is rigid if and only if no two distinct elements of $\mathcal{P}$ are comparable.

\section{RIGIDITY OF GRAPHS}

In this section we apply the results of Section 1 to study the rigidity of edge ideals of a graph.

Inseparable graphs. Let $G$ be a finite simple graph with vertex set $[n]$. The edge set of $G$ will be denoted by $E(G)$. Let $K$ be a field and $S=K\left[x_{1}, \ldots, x_{n}\right]$ the polynomial ring over $K$ in $n$ indeterminates $x_{1}, \ldots, x_{n}$. The edge ideal $I(G) \subseteq S$ of $G$ is defined to be the ideal generated by all products $x_{i} x_{j}$ with $\{i, j\} \in E(G)$. Let $\Delta(G)$ be the simplicial complex with $I(G)=I_{\Delta(G)}$ Then

$$
\Delta(G)=\{F \subseteq[n]: F \text { does not contain any edges of } G\},
$$

The simplicial complex $\Delta(G)$ is called the independence complex of $G$. The faces of $\Delta(G)$ are called the independent sets of $G$.

We call $G$ inseparable if $I(G)$ is inseparable. Let $i \in[n]$. Then $N(i)=\{j:\{j, i\} \in$ $E(G)\}$ is called the neighborhood of $i$. We denote by $G^{(i)}$ the complementary graph of the restriction $G_{N(i)}$ of $G$ to $N(i)$. In other words, $V\left(G^{(i)}\right)=N(i)$ and $E\left(G^{(i)}\right)=$ $\{\{j, k\}: j \neq k, j, k \in N(i)$ and $\{j, k\} \notin E(G)\}$. Note that $G^{(i)}$ is disconnected if and only if $N(i)=A \cup B$, where $A, B \neq \emptyset, A \cap B=\emptyset$ and all vertices of $A$ are adjacent to those of $B$.

Theorem 3.1. The following conditions are equivalent:

(a) The graph $G$ is inseparable;

(b) $G^{(i)}$ is connected for all $i$;

(c) $T^{1}(S / I(G))_{-\mathbf{e}_{i}}=0$ for all $i$.

Proof. We set $\Delta=\Delta(G)$. By Theorem 1.8, it suffices to prove that $G_{\{i\}}(\Delta)$ is connected if and only if $G^{(i)}$ is connected for each $i$. First we note that $V\left(G^{(i)}\right) \subseteq[n]$ and

$$
V\left(G_{\{i\}}(\Delta)\right)=\left\{F \subseteq[n]: F \text { is an independent set of } G \text { and } F \cap V\left(G^{(i)}\right) \neq \emptyset\right\} .
$$

Assume $G^{(i)}$ is connected. Given $F_{1}, F_{2} \in V\left(G_{\{i\}}(\Delta)\right)$, there exist $k_{1}, k_{2} \in V\left(G^{(i)}\right)$ with $k_{i} \in F_{i}$ for $i=1,2$. Suppose that $k_{1}=k_{2}$. Then $F_{1},\left\{k_{1}\right\}, F_{2}$ is a path in $G_{\{i\}}(\Delta)$, and thus $F_{1}$ is connected to $F_{2}$. Next suppose that $k_{1} \neq k_{2}$. Since $G^{(i)}$ is connected, there is a path $k_{1}=j_{0}, j_{1}, \ldots, j_{s}=k_{2}$ in $G^{(i)}$. Note that $\left\{j_{\ell}, j_{\ell+1}\right\} \in$ $V\left(G_{\{i\}}(\Delta)\right)$ for all $\ell=0, \ldots, s-1$. Therefore,

$$
F_{1},\left\{k_{1}\right\},\left\{j_{0}, j_{1}\right\},\left\{j_{1}\right\},\left\{j_{1}, j_{2}\right\},\left\{j_{2}\right\}, \ldots,\left\{j_{s-1}, j_{s}\right\},\left\{k_{2}\right\}, F_{2}
$$


is a path in $G_{\{i\}}(\Delta)$, and so $F_{1}$ is connected to $F_{2}$. It follows that $G_{\{i\}}(\Delta)$ is connected.

Conversely, assume that $G_{\{i\}}(\Delta)$ is connected. Given $k_{1}, k_{2} \in V\left(G^{(i)}\right)$, there is a path $\left\{k_{1}\right\}, F_{1}, F_{2}, \ldots, F_{2 t-1},\left\{k_{2}\right\}$ in $G_{\{i\}}(\Delta)$. Hence

$$
\left\{k_{1}\right\} \subseteq F_{1} \supseteq F_{2} \subseteq F_{3} \supseteq \ldots \subseteq F_{2 t-1} \supseteq\left\{k_{2}\right\} .
$$

We use the induction on $t$ to show that there is a path from $k_{1}$ to $k_{2}$ in $G^{(i)}$. If $t=1$ then $\left\{k_{1}, k_{2}\right\} \subseteq F_{1}$ and so $k_{1}$ is adjacent to $k_{2}$ (in $\left.G^{(i)}\right)$. For $t>1$, let $k_{0} \in F_{2} \cap V\left(G^{(i)}\right)$. Then $k_{1}$ is adjacent to $k_{0}$, and by induction hypothesis there is a path in $G^{(i)}$ from $k_{0}$ to $k_{2}$. Hence there is a path in $G^{(i)}$ from $k_{1}$ to $k_{2}$ and it follows that $G^{(i)}$ is connected.

Considering the proof of Theorem 3.1 one even shows that the graphs $G_{\{i\}}(\Delta)$ and $G^{(i)}$ have the same number of connected components.

Corollary 3.2. If $G$ contains no triangle, then $G$ is inseparable.

The conditions $(\alpha)$ and $(\beta)$. Let $G$ be a finite graph on the vertex set $[n]$ and $\Delta(G)$ be the independence complex of $G$. Let $A$ be a subset of $[n]$. Then link $A$ of $\Delta(G)$ can be interpreted as the independence complex of a suitable graph if $A$ is an independent subset of $G$. In order to show this we introduce some notation.

The set

$$
N(A)=\bigcup_{i \in A} N(i)
$$

is called the neighborhood of $A$ (in $G$ ), and the set

$$
N[A]=A \cup N(A)
$$

is called the closed neighborhood of $A$ (in $G$ ).

Let $B \subset[n]$. The induced subgraph of $G$ with vertex set $B$, is the graph $G_{B}$ with edges $\{i, j\} \in E(G)$ and such that $i, j \in B$. An induced cycle of $G$ is a cycle of $G$ which is of the form $G_{B}$. By $G \backslash A$ we denote the induced subgraph of $G$ on the vertex set $[n] \backslash A$.

Lemma 3.3. Let $A \subseteq[n]$ be an independent subset of $G$. Then $\operatorname{link}_{\Delta(G)} A$ is the independence complex of the graph $G \backslash N[A]$.

Proof. Note that $F \in \operatorname{link}_{\Delta(G)} A$ if and only if $F \subseteq[n] \backslash A$ and $F \cup A \in \Delta(G)$. The last condition is equivalent to saying that $F \cup A$ does not contain any edge of $G$. Thus $F \in \operatorname{link}_{\Delta(G)} A$ if and only if $F \subseteq[n] \backslash N[A]$ and $F$ does not contain any edge of $G$. Since the set of edges of $G$ in $[n] \backslash N(A)$ is the same as the set of edges of $G$ in $G \backslash N(A)$, the desired conclusion follows.

For a given subset $B \subseteq[n]$, we may easily express the sets

$$
\tilde{N}_{B}(\Delta(G)) \subseteq N_{B}(\Delta(G)) \subseteq[n]
$$

in terms of $G$ :

$$
N_{B}(\Delta(G))=\{F \subseteq[n]: F \cap B=\emptyset, F \text { contains no edges of } G \text {, but } F \cup B \text { does }\},
$$


and $\widetilde{N}_{B}(\Delta(G))=$

$\left\{F \in N_{B}(\Delta(G))\right.$ : there exists $B^{\prime} \subsetneq B$ such that $F \cup B^{\prime}$ contains an edge of $\left.G\right\}$.

The following lemma lists some obvious properties of these sets.

Lemma 3.4. Let $B \subseteq[n]$. Then the following statements hold:

(a) If $|B| \geq 3$ or $|B|=2$ and $B$ is not an edge of $G$, then $N_{B}(\Delta(G))=\widetilde{N}_{B}(\Delta(G))$.

(b) If $B$ is an edge, then $\emptyset \in N_{B}(\Delta(G))$, and $\widetilde{N}_{B}(\Delta(G))=\emptyset$ if and only $B$ is an isolated edge of $G$, i.e. it does not have a common vertex with any other edge of $G$.

Combining this lemma with Corollary 1.4 we obtain

Corollary 3.5. Let $\mathbf{b} \in\{0,1\}^{n}$ and let $B=\operatorname{supp} \mathbf{b}$. Suppose that $|B| \geq 2$. Then $T^{1}(\Delta(G))_{-\mathbf{b}}=0$ unless $B$ is an isolated edge in $G$. On the other hand, if $B$ is an isolated edge in $G$, then $T^{1}(\Delta(G))_{-\mathbf{b}}$ is one-dimensional.

Let $G$ be a graph on the vertex set $[n]$. Based on Theorem 3.1 and Corollary 3.5 we see that $\Delta(G)$ is $\emptyset$-rigid, (i.e., $T^{1}(\Delta(G))_{-\mathbf{b}}=0$ for every $\left.\mathbf{b} \in\{0,1\}^{n}\right)$ if and only if $G^{(i)}$ is connected for all $i \in[n]$ and $G$ contains no isolated edge. Combining this fact with Proposition 1.2 and Lemma 3.3 we obtain the following combinatorial conditions for a graph to be rigid: the graph $G$ is rigid if and only if for all independent sets $A \subseteq V(G)$ one has:

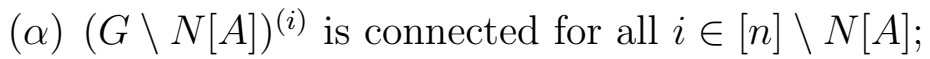

( $\beta) G \backslash N[A]$ contains no isolated edge.

It is obvious from Corollary 3.2 that any bipartite graph is inseparable and so it satisfies the condition $(\alpha)$, since any induced graph of a bipartite graph is again bipartite. But, by far, not all bipartite graphs are rigid. For example we have

Corollary 3.6. Let $G$ be a Cohen-Macaulay bipartite graph (i.e., $S / I(G)$ is CohenMacaulay). Then $G$ is not rigid.

Proof. By Proposition 2.3 we may assume that $G$ is connected. By 8 , Theorem 3.4] the graph $G$, after a suitable relabeling of its vertices, arises from a finite poset $P=\left\{p_{1}, \ldots, p_{n}\right\}$ as follows: $V(G)=\left\{p_{1}, \ldots, p_{n}, q_{1}, \ldots, q_{n}\right\}$ and $E(G)=$ $\left\{\left\{p_{i}, q_{j}\right\}: p_{i} \leq p_{j}\right\}$. We may assume that $p_{1}$ is a minimal element in $P$. Let $A=\left\{p_{2}, \ldots, p_{n}\right\}$. Then $N[A]=\left\{p_{2}, \ldots, p_{n}, q_{2}, \ldots, q_{n}\right\}$, and $G \backslash N[A]=\left\{p_{1}, q_{1}\right\}$. It follows from $(\beta)$ that $G$ is not rigid.

Recall that a vertex $v$ is called a free vertex if $\operatorname{deg} v=1$, and an edge $e$ is called a leaf if it has a free vertex. An edge $e$ of $G$ is called branch, if there exists a leaf $e^{\prime}$ with $e^{\prime} \neq e$ such that $e \cap e^{\prime} \neq \emptyset$.

Let $e=\{i, j\} \in E(G)$. We denote by $N_{0}(e)$ the set $(N(i) \cup N(j)) \backslash\{i, j\}$.

In the next proposition we present sufficient conditions for graph to satisfy $(\alpha)$ or $(\beta)$.

Proposition 3.7. Let $G$ be a finite graph on the vertex set $[n]$.

(a) Suppose that each vertex of a 3 -cycle in $G$ belongs to a leaf. Then $G$ satisfies $(\alpha)$, and hence $G$ is inseparable. In particular, if $G$ does not contain any 3 -cycle, then $G$ is inseparable. 
(b) Suppose that each edge of $G$ is a branch. Then $G$ satisfies $(\beta)$.

(c) Suppose that each edge of $G$ is a branch and each vertex of a 3-cycle of $G$ belongs to a leaf. Then $G$ is rigid.

Proof. (a) Suppose that $(\alpha)$ is not satisfied. Then there exists an independent set $A \subseteq[n]$ and $i \in[n] \backslash N[A]$ such that $N(i) \cap[n] \backslash N[A]=B \cup C$ with $B, C \neq \emptyset$ and $B \cap C=\emptyset$ and $\{j, k\} \in E(G \backslash N[A]) \subseteq E(G)$ for all $j \in B$ and all $k \in C$. Since $B, C \neq \emptyset$ there exist $j \in B$ and $k \in C$ such that $\{j, k\} \in E(G)$. Thus $i$ is a vertex of a 3-cycle in $G$. By assumption there exists a leaf $\{i, t\}$ in $G$. Suppose that $t \in N[A]$. If $t \in A$ then $i \in N[A]$, a contradiction. Thus $t \in N(A)$. Since $\operatorname{deg} t=1$ it follows that $i \in A$, again a contradiction. Therefore we see that $t \in[n] \backslash N[A]$. This implies that $t \in B \cup C$. We may assume that $t \in B$. So $\{t, k\} \in E(G)$, a contradiction since $N(t)=\{i\}$.

It follows from Theorem 3.1 that $G$ is inseparable if it satisfies $(\alpha)$. Suppose now that $G$ does not contain any 3 -cycle. Then, by the first part of the statement, $G$ satisfies condition $(\alpha)$, and so it is inseparable.

(b) Suppose that $G$ does not satisfy $(\beta)$. Then there exists an independent set $A$ of the vertices of $G$ such that $G \backslash N[A]$ contains an isolated edge, say $e=\{i, j\}$. We show that $e$ is not a branch of $G$ and so we get a contradiction. Let $v \in N_{0}(e)$ and let $\operatorname{deg} v=1$. Then we have either $N(v)=\{i\}$ or $N(v)=\{j\}$. Without loss of generality we may assume that $N(v)=\{i\}$. Since $e$ is an isolated edge in $G \backslash N[A]$ we have $v \in N[A]$. Suppose that $v \in A$. Then $\{i\}=N(v) \subseteq N(A)$, a contradiction. Thus $v \in N(A)$, and so there exists $t \in A$ such that $v \in N(t)$. Since $N(v)=\{i\}$ it follows that $t=i$. This implies that $i \in A$, which is again a contradiction. Hence $\operatorname{deg} v \geq 2$, as desired.

(c) follows from (a) and (b).

Rigid graphs. The next two lemmata will help us to classify the rigid chordal graphs and rigid graphs without induced cycles of length 4,5 or 6 .

Recall that a graph $G$ is chordal if any cycle of length $\geq 4$ has chord. A chord of a cycle $C$ is an edge $\{i, j\}$ of $G$ with $i, j \in V(C)$ which is not an edge of $C$

Lemma 3.8. Let $G$ be a rigid graph on the vertex set $[n]$, and let $A$ be an independent set of vertices of $G$. Then $G \backslash N[A]$ is rigid.

Proof. Let $B$ be an independent set of vertices of $G \backslash N[A]$. Then $A \cup B$ is an independent set of vertices of $G$. Indeed, suppose that $\{i, j\} \in E(G)$ for some $i \in A$ and some $j \in B$. Then $j \in N(i)$ implies that $j \in N(A) \subseteq N[A]$. Since $N[A] \cap B=\emptyset$ it follows that $j \notin B$ which is a contradiction.

Clearly, $N[A] \cup N[B]=N[A \cup B]$. Thus, $([n] \backslash N[A]) \backslash N[B]=[n] \backslash N[A \cup B]$. Since for any subset $C$ of $[n], G \backslash C$ is an induced subgraph of $G$, we have $(G \backslash N[A]) \backslash N[B]=$ $G \backslash N[A \cup B]$. Since rigidity is characterized by $(\alpha)$ and $(\beta)$, the statement follows. 
Let $G$ be a graph on the vertex set $[n]$. For each $e=\{i, j\} \in E(G)$ we define the set $O_{G}(e)$ as follows:

$$
O_{G}(e)=\left\{v^{\prime}: v^{\prime} \in \bigcup_{v \in N_{0}(e)} N(v), N\left(v^{\prime}\right) \cap\{i, j\}=\emptyset\right\} .
$$

Lemma 3.9. Let $G$ be a rigid graph on the vertex set $[n]$ which does not contain any induced 4-cycle, and let $e=\{i, j\}$ be an edge of $G$ which is not a branch. Then $O_{G}(e) \neq \emptyset$.

Proof. First we show that for all $v \in N_{0}(e)$ we have $N(v) \backslash\{i, j\} \neq \emptyset$. Note that since $G$ is rigid the edge $e$ is not isolated, and so $N_{0}(e) \neq \emptyset$. Suppose that there exists $v \in N_{0}(e)$ such that $N(v) \subseteq\{i, j\}$. Without loss of generality we may assume that $v \in N(i)$. Since $e$ is not a branch we have $\operatorname{deg} v \geq 2$. It follows that $N(v)=\{i, j\}$. Therefore $G^{(v)}$ consists of two isolated vertices $i$ and $j$ which contradicts the fact that $G$ is rigid.

Now suppose that $O_{G}(e)=\emptyset$, i.e., for all $v \in N_{0}(e)$ and for all $v^{\prime} \in N(v)$ we have $N\left(v^{\prime}\right) \cap\{i, j\} \neq \emptyset$. Since $N_{0}(e) \neq \emptyset$ we may assume that there exists $v \in N(i)$ with $v \neq j$. As shown above $N(v) \backslash\{i, j\} \neq \emptyset$. Suppose that for all $v^{\prime} \in N(v) \backslash\{i, j\}$ we have $i \in N\left(v^{\prime}\right)$. Then $i$ is an isolated vertex in $G^{(v)}$, a contradiction. Thus there exists $v^{\prime} \in N(v) \backslash\{i, j\}$ such that $v^{\prime} \notin N(i)$. Hence $v^{\prime} \in N(j)$ which implies that $v \in N(j)$ because $G$ does not contain any induced 4-cycle. Since $v^{\prime} \in N(j)$ and $v^{\prime} \neq i, j$ we have $v^{\prime} \in N_{0}(e)$. So $N\left(v^{\prime \prime}\right) \cap\{i, j\} \neq \emptyset$ for all $v^{\prime \prime} \in N\left(v^{\prime}\right)$. As shown above $N\left(v^{\prime}\right) \backslash\{i, j\} \neq \emptyset$. Suppose that there exists $v^{\prime \prime} \in N\left(v^{\prime}\right) \backslash\{i, j\}$ such that $v^{\prime \prime} \notin N(j)$. Then $v^{\prime \prime} \in N(i)$. It follows that $G$ contains the induced 4-cycle with vertices $i, j, v^{\prime}$ and $v^{\prime \prime}$, a contradiction. Consequently, $v^{\prime \prime} \in N(j)$ for all $v^{\prime \prime} \in N\left(v^{\prime}\right) \backslash\{i, j\}$. Then $j$ is an isolated vertex in $G^{\left(v^{\prime}\right)}$, a contradiction. This completes the proof.

Theorem 3.10. Let $G$ be a graph on the vertex set $[n]$ such that $G$ does not contain any induced cycle of length 4, 5 or 6 . Then $G$ is rigid if and only if each edge of $G$ is a branch and each vertex of a 3-cycle of $G$ belongs to a leaf.

Proof. By using part (c) of Proposition 3.7 it is enough to show that if a rigid graph $G$ does not contain any induced cycle of length 4,5 or 6 , then each edge of $G$ is a branch and each vertex of a 3-cycle of $G$ belongs to a leaf.

Suppose that $e=\{i, j\} \in E(G)$ is not a branch. By Lemma [3.9. $O_{G}(e) \neq \emptyset$. We claim that there exists $A \subseteq O_{G}(e)$ such that $A$ is independent in $G$ and $e$ is an isolated edge in $G \backslash N[A]$. This will imply that $G$ is not rigid, a contradiction. Let $G^{\prime}$ be the induced subgraph of $G$ on the vertex set $O_{G}(e)$ and let $C_{1}, \ldots, C_{m}$ be the connected components of $G^{\prime}$. Let $u, v \in V\left(C_{k}\right)$ such that $\{u, v\} \in E(G)$. We show that either $N(u) \cap N_{0}(e) \subseteq N(v) \cap N_{0}(e)$ or $N(v) \cap N_{0}(e) \subseteq N(u) \cap N_{0}(e)$.

Assume that $N(u) \cap N_{0}(e) \nsubseteq N(v) \cap N_{0}(e)$. Then there exists $x \in N(u) \cap N_{0}(e)$ such that $\{v, x\}$ is not an edge in $G$. Without loss of generality we may assume that $i \in N(x)$.

Let $y \in N(v) \cap N_{0}(e)$, and first suppose that $y \in N(i)$. Then we have the 5 -cycle with vertices $i, x, u, v$ and $y$. Since $v, u \in O_{G}(e)$ it follows that $\{u, i\},\{v, i\} \notin E(G)$ and since $\{v, x\} \notin E(G)$ it follows that $\{u, y\} \in E(G)$ because $G$ does not contain 
any induced cycle of length 4 and 5. Therefore $y \in N(u) \cap N_{0}(e)$. On the other hand if $y \in N(j)$, then we have the 6 -cycle with vertices $i, x, u, v, y$ and $j$. Note that $\{v, x\},\{u, i\},\{u, j\},\{v, i\},\{v, j\} \notin E(G)$. This implies that $\{v, y\} \in E(G)$ since $G$ does not contain any induced cycle of length 4, 5 and 6 . Thus either $N(u) \cap N_{0}(e) \subseteq N(v) \cap N_{0}(e)$ or $N(v) \cap N_{0}(e) \subseteq N(u) \cap N_{0}(e)$, as desired.

Now given $C_{k}$ we choose a maximal set $D_{k}=\left\{u_{1}, \ldots, u_{l}\right\} \subseteq V\left(C_{k}\right)$ with the property that the sets $N\left(u_{r}\right) \cap N_{0}(e)$ are pairwise different. After having defined the set $D_{k}$ for each $C_{k}$ we are ready to define the set $A$.

We let $A$ be the unique subset of $O_{G}(e)$ such that $A \cap C_{k}$ consists of all elements $u_{r} \in D_{k}$ with the property that $N\left(u_{r}\right) \cap N_{0}(e) \nsubseteq N\left(u_{s}\right) \cap N_{0}(e)$ for all $u_{s} \in D_{k}$ with $s \neq r$.

In order to complete the proof we show that $A$ is independent in $G$ and $e$ is an isolated edge in $G \backslash N[A]$. Let $u, v \in a$ and assume that $\{u, v\} \in E(G)$. Then there exists $k$ such that $u, v \in D_{k} \subseteq V\left(C_{k}\right)$. Therefore either $N(u) \cap N_{0}(e) \subseteq N(v) \cap N_{0}(e)$ or $N(v) \cap N_{0}(e) \subseteq N(u) \cap N_{0}(e)$. Thus by the choice of $A$, it follows that $u=v$, a contradiction. So $A$ is an independent set of $G$.

Finally we show that $e$ is an isolated edge of $G^{\prime \prime}:=G \backslash N[A]$. Note that for any $v \in N_{0}(e) \cap V\left(G^{\prime \prime}\right)$ and for any $v^{\prime} \in N(v) \backslash\{i, j\}$ we have $N\left(v^{\prime}\right) \cap\{i, j\} \neq \emptyset$. In fact, suppose that there exists $v_{1} \in N(v)$ such that $N\left(v_{1}\right) \cap\{i, j\}=\emptyset$. So $v_{1} \in V\left(C_{k}\right)$ for some $k$. If $v_{1} \in A$, then $v \in N(A) \subseteq N[A]$, a contradiction. Hence $v_{1} \notin A$. Therefore, by the choice of $A$, there exists $v_{2} \in A$ such that $N\left(v_{1}\right) \cap N_{0}(e) \subseteq$ $N\left(v_{2}\right) \cap N_{0}(e)$. Since $v \in N\left(v_{1}\right) \cap N_{0}(e)$ we have $v \in N\left(v_{2}\right)$ and so $v \in N(A) \subseteq N[A]$, a contradiction. This shows that $O_{G^{\prime \prime}}(e)=\emptyset$.

Suppose that $e$ is not an isolated edge of $G^{\prime \prime}$, i.e., $N_{0}(e) \cap V\left(G^{\prime \prime}\right) \neq \emptyset$. We observe that $e$ is not a branch in $G^{\prime \prime}$. Indeed, if $e$ is a branch, then since $e$ is not isolated, there exists $v \in N_{0}(e) \cap V\left(G^{\prime \prime}\right)$ such that degree of $v$ in $G^{\prime \prime}$ is one. We may assume that $v \in N(i) \backslash N(j)$. Since $e$ is not a branch in $G$ we have $N(v) \backslash\{i, j\} \neq \emptyset$ and for any $v^{\prime} \in N(v) \backslash\{i, j\}$ we have $v^{\prime} \in N[A]$. This implies that for any $v^{\prime} \in N(v)$, $v^{\prime} \in N(A)$ because if $v^{\prime} \in a$, then $v \in N(A) \subseteq N[A]$ and hence $v \notin V\left(G^{\prime \prime}\right)$, a contradiction. As seen in the previous paragraph, for any $v^{\prime} \in N(v) \backslash\{i, j\}$ we have $N\left(v^{\prime}\right) \cap\{i, j\} \neq \emptyset$. If $v^{\prime} \notin N(i)$, then $v^{\prime} \in N(j)$. Since $v \notin N(j)$ we will get the induced 4-cycle with the vertices $i, v, v^{\prime}$ and $j$, a contradiction. So $v^{\prime} \in N(i)$ for any $v^{\prime} \in N(v)$. It follows that $i$ is an isolated vertex in $G^{(v)}$, a contradiction. Thus $e$ is not a branch in $G^{\prime \prime}$.

Lemma 3.8 implies that $G^{\prime \prime}$ is rigid and hence by Lemma 3.9] it follows that $O_{G^{\prime \prime}}(e) \neq \emptyset$, a contradiction. So indeed $e$ is isolated in $G^{\prime \prime}$.

Now we prove that each vertex of a 3-cycle of $G$ belongs to a leaf. Suppose that there exists $i \in[n], i$ belongs to a 3-cycle in $G$ and it does not belong to a leaf. So for all $v \in N(i)$ we have $\operatorname{deg} v \geq 2$. Let $j$ and $k$ be the two other vertices of this 3-cycle. If $N(i)=\{j, k\}$, then $j$ and $k$ are isolated vertices of $G^{(i)}$, contradicting $(\alpha)$. So $N(i) \backslash\{j, k\} \neq \emptyset$. Since each edge of the graph $G$ is a branch, for any $v \in N(i)$ the edge $\{i, v\}$ is a branch. Since $i$ does not belong to a leaf it follows that any $v \in N(i)$ belongs to a leaf. Thus for any $v \in N(i)$ there exists $i_{v} \in N(v)$ with $\operatorname{deg} i_{v}=1$. Set $a=\bigcup_{v \in N(i) \backslash\{j, k\}}\left\{i_{v}\right\}$. Clearly, $A$ is an independent set of the vertices of $G$ and $j, k$ 
are two isolated vertices in $(G \backslash N[A])^{(i)}$, a contradiction. Consequently, $i$ belongs to a leaf, as desired.

Corollary 3.11. Let $G$ be a chordal graph. Then $G$ is rigid if and only if each edge of $G$ is a branch and each vertex of a 3-cycle of $G$ belongs to a leaf.

Corollary 3.12. Let $G$ be a graph on the vertex set $[n]$. Suppose that all cycles of $G$ have length $\geq 7$ (which for example is the case when $G$ is a forest). Then $G$ is rigid if and only if each edge of $G$ is a branch.

Proof. Since $G$ does not contain any 3-cycle, the statement follows from Theorem 3.10 .

As another application we have

Corollary 3.13. Let $C$ be a cycle. Then $C$ is rigid if and only if $C$ is a 4-cycle or a 6-cycle.

Proof. Suppose that $|C| \neq 4,5,6$. Then by Theorem 3.10 , $C$ is not rigid. Suppose now that $|C|=5$. Then $C \backslash N[A]$ is an isolated edge, where $A=\{i\}$ for some vertex $i$ of $C$, and hence the condition $(\beta)$ is not satisfied. So $C$ is not rigid also when $|C|=5$. In conclusion, $C$ is not rigid if $|C| \notin\{4,6\}$.

Next suppose that $|C| \in\{4,6\}$. Since $C$ does not contain a 3-cycle, it follows that $C$ satisfies $(\alpha)$ by Proposition 3.7 (a).

Note that for any nonempty independent subset $A$ of $V(C), C \backslash N[A]$ is either an empty graph (i.e., a graph containing no edge) or a path of length 3 . Therefore, the cycle $C$ also satisfies the condition $(\beta)$. Hence $C$ is rigid.

Acknowledgement: We would like to express our deep gratitude to the referee for his/her careful reading and for his/her excellent advices, which improved the presentation of this paper considerably.

\section{REFERENCES}

[1] K. Altmann, J.A. Christophersen, Deforming Stanley-Reisner schemes, Math. Ann. 348(3) (2010), 513-537.

[2] K. Altmann, J.A. Christophersen, Cotangent cohomology of Stanley-Reisner rings. Manuscripta Math. 115(3) (2004), 361-378.

[3] V. Ene, J. Herzog, F. Mohammadi, Monomial ideals and toric rings of Hibi type arising from a finite poset, European Journal of Combinatorics 32(3) (2011), 404-421.

[4] G. Fløystad, B.M. Greve, J. Herzog, Letterplace and co-letterplace ideals of posets, http://arxiv.org/abs/1501.04523v1

[5] D. Eisenbud, Commutative Algebra with a View Toward Algebraic Geomtry, Springer, 1994.

[6] J. Herzog, Deformation von Cohen-Macaulay Algebren, Journal für die reine und angewandte Mathematik 318 (1980), 83-105.

[7] J. Herzog, and T. Hibi, Monomial Ideals, Graduate Text in Mathematics, Springer, 2011.

[8] J. Herzog, T. Hibi, Distributive lattices, bipartite graphs and Alexander duality, J. Algebraic Combin. 22(3) (2005), 289-302.

[9] S. Lichtenbaum, M. Schlessinger, The Cotangent Complex of a Morphism, Trans. Amer. Math. Soc. 128(1) (1967), 41-70.

[10] J. Stevens, Deformations of singularities, Lecture Notes in Mathematics (1811), Berlin: Springer, 2003. 
Klaus Altmann, Institut für Mathematik, Freie Universität Berlin, Arnimallee 3, D-14195 Berlin, Germany

Email address: altmann@math.fu-berlin.de

Mina Bigdeli, Department of Mathematics, Institute for Advanced Studies in Basic Sciences (IASBS), Gava ZANG, 45195-1159 Zanjan, 45137-66731 Iran

Email address: m.bigdelie@iasbs.ac.ir

Jürgen Herzog, Fakultät für Mathematik, Universität Duisburg-Essen, 45117 Essen, Germany

Email address: juergen.herzog@gmail.com

Dancheng Lu, Department of Mathematics, Soochow University, 215006 Suzhou, P.R.CHINA

Email address: ludancheng@suda.edu.cn 